\title{
Multiparty Compatibility in Communicating Automata: Characterisation and Synthesis of Global Session Types
}

\author{
Pierre-Malo Deniélou ${ }^{1}$ and Nobuko Yoshida ${ }^{2}$ \\ ${ }^{1}$ Royal Holloway, University of London $\quad{ }^{2}$ Imperial College London
}

\begin{abstract}
Multiparty session types are a type system that can ensure the safety and liveness of distributed peers via the global specification of their interactions. To construct a global specification from a set of distributed uncontrolled behaviours, this paper explores the problem of fully characterising multiparty session types in terms of communicating automata. We equip global and local session types with labelled transition systems (LTSs) that faithfully represent asynchronous communications through unbounded buffered channels. Using the equivalence between the two LTSs, we identify a class of communicating automata that exactly correspond to the projected local types. We exhibit an algorithm to synthesise a global type from a collection of communicating automata. The key property of our findings is the notion of multiparty compatibility which non-trivially extends the duality condition for binary session types.
\end{abstract}

\section{Introduction}

Over the last decade, session types $[16,24]$ have been studied as data types or functional types for communications and distributed systems. A recent discovery by $[6,26]$, which establishes a Curry-Howard isomorphism between binary session types and linear logics, confirms that session types and the notion of duality between type constructs have canonical meanings. On the practical side, multiparty session types [3,17] were proposed as a major generalisation of binary session types. It can enforce communication safety and deadlock-freedom for more than two peers thanks to a choreographic specification (called global type) of the interaction. Global types are projected to end-point types (called local types), against which processes can be statically type-checked and verified to behave correctly.

The motivation of this paper comes from our practical experiences that, in many situations, even where we start from the end-point projections of a choreography, we need to reconstruct a global type from distributed specifications. End-point specifications are usually available, either through inference from the control flow, or through existing service interfaces, and always in forms akin to individual communicating finite state machines. If one knows the precise conditions under which a global type can be constructed (i.e. the conditions of synthesis), not only the global safety property which multiparty session types ensure is guaranteed, but also the generated global type can be used as a refinement and be integrated within the distributed system development life-cycle (see $\S 5$ for applications $[22,23]$ ). This paper attempts to give the synthesis condition as a sound and complete characterisation of multiparty session types with respect to Communicating Finite State Machines (CFSMs) [5]. CFSMs have been a wellstudied formalism for analysing distributed safety properties and are widely present in industry tools. They can been seen as generalised end-point specifications, therefore, an excellent target for a common comparison ground and for synthesis. As explained below, to identify a complete set of CFSMs for synthesis, we first need to answer a question - what is the canonical duality notion in multiparty session types? 
Characterisation of binary session types as communicating automata The subclass which fully characterises binary session types was actually proposed by Gouda, Manning and Yu in 1984 [15] in a pure communicating automata context. ${ }^{1}$ Consider a simple business protocol between a Buyer and a Seller from the Buyer's viewpoint: Buyer sends the title of a book, Seller answers with a quote. If Buyer is satisfied by the quote, then he sends his address and Seller sends back the delivery date; otherwise it retries the same conversation. This can be described by the following session type:

$$
\mu \mathrm{t} \text {.! title; ?quote; !\{ ok:!addrs; ?date; end, retry : t \} }
$$

where the operator ! title denotes an output of the title, whereas ?quote denotes an input of a quote. The output choice features the two options ok and retry and ; denotes sequencing. end represents the termination of the session, and $\mu \mathrm{t}$ is recursion.

The simplicity and tractability of binary sessions come from the notion of duality in interactions [14]. The interaction pattern of the Seller is fully given as the dual of the type in (1.1) (exchanging input ! and output? in the original type). When composing two parties, we only have to check they have mutually dual types, and the resulting communication is guaranteed to be deadlock-free. Essentially the same characterisation is given in communicating automata. Buyer and Seller's session types are represented by the following two machines.

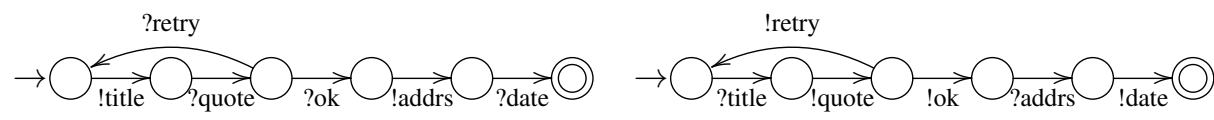

We can observe that these CFSMs satisfy three conditions. First, the communications are deterministic: messages that are part of the same choice, ok and retry here, are distinct. Secondly, there is no mixed state (each state has either only sending actions or only receiving actions). Third, these two machines have compatible traces (i.e. dual): the Seller machine can be defined by exchanging sending to receiving actions and vice versa. Breaking one of these conditions allows deadlock situations and breaking one of the first two conditions makes the compatibility checking undecidable [15].

Multiparty compatibility This notion of duality is no longer effective in multiparty communications, where the whole conversation cannot be reconstructed from only a single behaviour. To bypass the gap between binary and multiparty, we take the synthesis approach, that is to find conditions which allow a global choreography to be built from the local machine behaviour. Instead of directly trying to decide whether the communications of a system will satisfy safety (which is undecidable in the general case), inferring a global type guarantees the safety as a direct consequence.
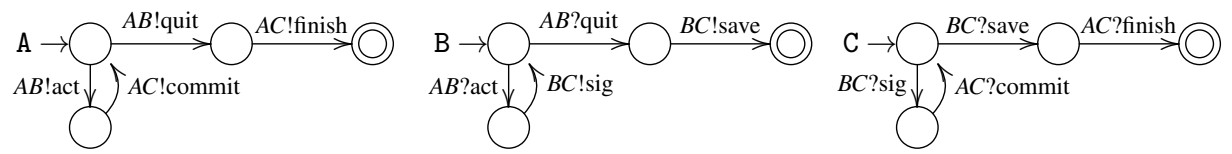

Fig. 1. Commit example: CFSMs

We give a simple example to illustrate the problem. The commit protocol in Figure 1 involves three machines: Alice A, Bob B and Carol C. A orders B to act or quit. If act is sent, $B$ sends a signal to $C$, and $A$ sends a commitment to $C$ and continues. Otherwise $B$ informs $\mathrm{C}$ to save the data and $\mathrm{A}$ gives the final notification to $\mathrm{C}$ to terminate the protocol.

${ }^{1}$ Villard [25] independently found this subset in the context of channel contracts [12]. 
This paper presents a decidable notion of multiparty compatibility as a generalisation of duality of binary sessions, which in turns characterises a synthesis condition. The idea is to check the duality between each automaton and the rest, up to the internal communications (1-bounded executions in the terminology of CFSMs, see $\S 2$ ) that the other machines will independently perform. For example, in Figure 1, to check the compatibility of trace $B C$ ?sig $A C$ ? commit in $\mathrm{C}$, we execute the internal communications between $\mathrm{A}$ and $\mathrm{B}$ such that $A B$ !act $\cdot A B$ ?act and observes the dual trace $B C$ !sig $\cdot A C$ !commit from $B$ and $A$. If this extended duality is valid for all the machines from any 1-bounded reachable state, then they satisfy multiparty compatibility and can build a well-formed global choreography.

Contributions and Outline Section 3 defines new labelled transition systems for global and local types that represent the abstract observable behaviour of typed processes. We prove that a global type behaves exactly as its projected local types, and the same result between a single local type and its CFSMs interpretation. These correspondences are the key to prove the main theorems. Section 4 defines multiparty compatibility, studies its safety and liveness properties, gives an algorithm for the synthesis of global types from CFSMs, and proves the soundness and completeness results between global types and CFSMs. Section 5 discusses related work and concludes. The full proofs can be found in Appendix.

In Appendix $\mathrm{C}$, we also extend our result to generalised multiparty session types, a recent class of multiparty session types [11] with graph-like control flow and parallelism. The same multiparty compatibility as in $\S 4$ can be used without modification, although well-formedness condition need to be generalised. The synthesis algorithm relies on Petri net intermediate representations [9] and 1-bounded behavioural exploration. Our result is applicable to generate a core part of Choreography BPMN 2.0 specification [4] from CFSMs.

\section{Communicating Finite State Machines}

This section starts from some preliminary notations (following [8]). $\varepsilon$ is the empty word. $\mathbb{A}$ is a finite alphabet and $\mathbb{A}^{*}$ is the set of all finite words over $\mathbb{A} .|x|$ is the length of a word $x$ and $x . y$ or $x y$ the concatenation of two words $x$ and $y$. Let $\mathcal{P}$ be a set of participants fixed throughout the paper: $\mathcal{P} \subseteq\{\mathrm{A}, \mathrm{B}, \mathrm{C}, \ldots, \mathrm{p}, \mathrm{q}, \ldots\}$.

Definition 2.1 (CFSM). A communicating finite state machine is a finite transition system given by a 5 -tuple $M=\left(Q, C, q_{0}, \mathbb{A}, \delta\right)$ where (1) $Q$ is a finite set of states; (2) $C=\left\{\mathrm{pq} \in \mathcal{P}^{2} \mid \mathrm{p} \neq \mathrm{q}\right\}$ is a set of channels; (3) $q_{0} \in Q$ is an initial state; (4) $\mathbb{A}$ is a finite alphabet of messages, and (5) $\delta \subseteq Q \times(C \times\{!, ?\} \times \mathbb{A}) \times Q$ is a finite set of transitions.

In transitions, $\mathrm{pq}$ ! $a$ denotes the sending action of $a$ from process $\mathrm{p}$ to process $\mathrm{q}$, and pq? $a$ denotes the receiving action of $a$ from p by q. $\ell, \ell^{\prime}$ range over actions and we define the subject of an action $\ell$ as the principal in charge of it: $\operatorname{subj}(\mathrm{pq} ! a)=\operatorname{subj}(\mathrm{qp} ? a)=\mathrm{p}$.

A state $q \in Q$ whose outgoing transitions are all labelled with sending (resp. receiving) actions is called a sending (resp. receiving) state. A state $q \in Q$ which does not have any outgoing transition is called final. If $q$ has both sending and receiving outgoing transitions, $q$ is called mixed. We say $q$ is directed if it contains only sending (resp. receiving) actions to (resp. from) the same participant. A path in $M$ is a finite sequence of $q_{0}, \ldots, q_{n}(n \geq 1)$ such that $\left(q_{i}, \ell, q_{i+1}\right) \in \delta(0 \leq i \leq n-1)$, and we write $q \stackrel{\ell}{\rightarrow} q^{\prime}$ if 
$\left(q, \ell, q^{\prime}\right) \in \delta . M$ is connected if for every state $q \neq q_{0}$, there is a path from $q_{0}$ to $q$. Hereafter we assume each CFSM is connected.

A CFSM $M=\left(Q, C, q_{0}, \mathbb{A}, \delta\right)$ is deterministic if for all states $q \in Q$ and all actions $\ell,\left(q, \ell, q^{\prime}\right),\left(q, \ell, q^{\prime \prime}\right) \in \delta$ imply $q^{\prime}=q^{\prime \prime} .^{2}$

Definition 2.2 (CS). A (communicating) system $S$ is a tuple $S=\left(M_{\mathrm{p}}\right)_{\mathrm{p} \in \mathcal{P}}$ of CFSMs such that $M_{\mathrm{p}}=\left(Q_{\mathrm{p}}, C, q_{0 \mathrm{p}}, \mathbb{A}, \delta_{\mathrm{p}}\right)$.

For $M_{\mathrm{p}}=\left(Q_{\mathrm{p}}, C, q_{0 \mathrm{p}}, \mathbb{A}, \delta_{\mathrm{p}}\right)$, we define a configuration of $S=\left(M_{\mathrm{p}}\right)_{\mathrm{p} \in \mathcal{P}}$ to be a tuple $s=(\vec{q} ; \vec{w})$ where $\vec{q}=\left(q_{\mathrm{p}}\right)_{\mathrm{p} \in \mathcal{P}}$ with $q_{\mathrm{p}} \in Q_{\mathrm{p}}$ and where $\vec{w}=\left(w_{\mathrm{pq}}\right)_{\mathrm{p} \neq \mathrm{q} \in \mathcal{P}}$ with $w_{\mathrm{pq}} \in \mathbb{A}^{*}$. The element $\vec{q}$ is called a control state and $q \in Q_{i}$ is the local state of machine $M_{i}$.

Definition 2.3 (reachable state). Let $S$ be a communicating system. A configuration $s^{\prime}=\left(\vec{q}^{\prime} ; \vec{w}^{\prime}\right)$ is reachable from another configuration $s=(\vec{q} ; \vec{w})$ by the firing of the transition $t$, written $s \rightarrow s^{\prime}$ or $s^{t} \rightarrow s^{\prime}$, if there exists $a \in \mathbb{A}$ such that either:

1. $t=\left(q_{\mathrm{p}}, \mathrm{pq} ! a, q_{\mathrm{p}}^{\prime}\right) \in \delta_{\mathrm{p}}$ and (a) $q_{\mathrm{p}^{\prime}}^{\prime}=q_{\mathrm{p}^{\prime}}$ for all $\mathrm{p}^{\prime} \neq \mathrm{p}$; and (b) $w_{\mathrm{pq}}^{\prime}=w_{\mathrm{pq}} \cdot a$ and $w_{\mathrm{p}^{\prime} \mathrm{q}^{\prime}}^{\prime}=w_{\mathrm{p}^{\prime} \mathrm{q}^{\prime}}$ for all $\mathrm{p}^{\prime} \mathrm{q}^{\prime} \neq \mathrm{pq}$; or

2. $t=\left(q_{\mathrm{q}}, \mathrm{pq}\right.$ ? $\left.a, q_{\mathrm{q}}^{\prime}\right) \in \delta_{\mathrm{q}}$ and (a) $q_{\mathrm{p}^{\prime}}^{\prime}=q_{\mathrm{p}^{\prime}}$ for all $\mathrm{p}^{\prime} \neq \mathrm{q}$; and (b) $w_{\mathrm{pq}}=a . w_{\mathrm{pq}}^{\prime}$ and $w_{\mathrm{p}^{\prime} \mathrm{q}^{\prime}}^{\prime}=w_{\mathrm{p}^{\prime} \mathrm{q}^{\prime}}$ for all $\mathrm{p}^{\prime} \mathrm{q}^{\prime} \neq \mathrm{pq}$.

The condition (1-b) puts the content $a$ to a channel pq, while (2-b) gets the content $a$ from a channel pq. The reflexive and transitive closure of $\rightarrow$ is $\rightarrow^{*}$. For a transition $t=\left(s, \ell, s^{\prime}\right)$, we refer to $\ell$ by act $(t)$. We write $s_{1} \stackrel{t_{1} \cdots t_{m}}{\longrightarrow} s_{m+1}$ for $s_{1} \stackrel{t_{1}}{\longrightarrow} s_{2} \cdots \stackrel{t_{m}}{\rightarrow} s_{m+1}$ and use $\varphi$ to denote $t_{1} \cdots t_{m}$. We extend act to these sequences: $\operatorname{act}\left(t_{1} \cdots t_{n}\right)=\operatorname{act}\left(t_{1}\right) \cdots \operatorname{act}\left(t_{n}\right)$.

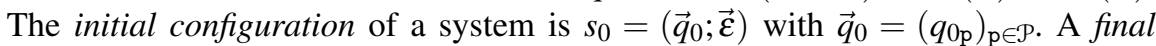
configuration of the system is $s_{f}=(\vec{q} ; \vec{\varepsilon})$ with all $q_{\mathrm{p}} \in \vec{q}$ final. A configuration $s$ is reachable if $s_{0} \rightarrow^{*} s$ and we define the reachable set of $S$ as $R S(S)=\left\{s \mid s_{0} \rightarrow{ }^{*} s\right\}$. We define the traces of a system $S$ to be $\operatorname{Tr}(S)=\left\{\operatorname{act}(\varphi) \mid \exists s \in R S(S), s_{0} \underline{\varphi}_{\rightarrow} s\right\}$.

We now define several properties about communicating systems and their configurations. These properties will be used in $\S 4$ to characterise the systems that correspond to multiparty session types. Let $S$ be a communicating system, $t$ one of its transitions and $s=(\vec{q} ; \vec{w})$ one of its configurations. The following definitions of configuration properties follow [8, Definition 12].

1. $s$ is stable if all its buffers are empty, i.e., $\vec{w}=\vec{\varepsilon}$.

2. $s$ is a deadlock configuration if $s$ is not final, and $\vec{w}=\vec{\varepsilon}$ and each $q_{\mathrm{p}}$ is a receiving state, i.e. all machines are blocked, waiting for messages.

3. $s$ is an orphan message configuration if all $q_{\mathrm{p}} \in \vec{q}$ are final but $\vec{w} \neq \emptyset$, i.e. there is at least an orphan message in a buffer.

4. $s$ is an unspecified reception configuration if there exists $\mathrm{q} \in \mathcal{P}$ such that $q_{\mathrm{q}}$ is a receiving state and $\left(q_{\mathrm{q}}, \mathrm{pq} ? a, q_{\mathrm{q}}^{\prime}\right) \in \delta$ implies that $\left|w_{\mathrm{pq}}\right|>0$ and $w_{\mathrm{pq}} \notin a \mathbb{A}^{*}$, i.e $q_{\mathrm{q}}$ is prevented from receiving any message from buffer $\mathrm{pq}$.

A sequence of transitions is said to be $k$-bounded if no channel of any intermediate configuration $s_{i}$ contains more than $k$ messages. We define the $k$-reachability set of $S$ to be the largest subset $R S_{k}(S)$ of $R S(S)$ within which each configuration $s$ can be

\footnotetext{
2 "Deterministic" often means the same channel should carry a unique value, i.e. if $\left(q, c ! a, q^{\prime}\right) \in$ $\delta$ and $\left(q, c ! a^{\prime}, q^{\prime \prime}\right) \in \delta$ then $a=a^{\prime}$ and $q^{\prime}=q^{\prime \prime}$. Here we follow a different definition [8] in order to represent branching type constructs.
} 
reached by a $k$-bounded execution from $s_{0}$. Note that, given a communicating system $S$, for every integer $k$, the set $R S_{k}(S)$ is finite and computable. We say that a trace $\varphi$ is $n$-bound, written bound $(\varphi)=n$, if the number of send actions in $\varphi$ never exceeds the number of receive actions by $n$. We then define the equivalences: (1) $S \approx S^{\prime}$ is $\forall \varphi, \varphi \in$ $\operatorname{Tr}(S) \Leftrightarrow \varphi \in \operatorname{Tr}\left(S^{\prime}\right)$; and (2) $S \approx_{n} S^{\prime}$ is $\forall \varphi$, bound $(\varphi) \leq n \Rightarrow\left(\varphi \in \operatorname{Tr}(S) \Leftrightarrow \varphi \in \operatorname{Tr}\left(S^{\prime}\right)\right)$.

The following key properties will be examined throughout the paper as properties that multiparty session type can enforce. They are undecidable in general CFSMs.

Definition 2.4 (safety and liveness). (1) A communicating system $S$ is deadlock-free (resp. orphan message-free, reception error-free) if for all $s \in R S(S)$, $s$ is not a deadlock (resp. orphan message, unspecified reception) configuration. (2) $S$ satisfies the liveness property $^{3}$ if for all $s \in R S(S)$, there exists $s \longrightarrow^{*} s^{\prime}$ such that $s^{\prime}$ is final.

\section{Global and local types: the LTSs and translations}

This section presents the multiparty session types, our main object of study. For the syntax of types, we follow [3] which is the most widely used syntax in the literature. We introduce two labelled transition systems, for local types and for global types, and show the equivalence between local types and communicating automata.

Syntax A global type, written $G, G^{\prime}, .$. , describes the whole conversation scenario of a multiparty session as a type signature, and a local type, written by $T, T^{\prime}, \ldots$, typeabstract sessions from each end-point's view. p, $q, \cdots \in \mathcal{P}$ denote participants (see $\S 2$ for conventions). The syntax of types is given as:

$$
\begin{aligned}
& G::=\mathrm{p} \rightarrow \mathrm{p}^{\prime}:\left\{a_{j} \cdot G_{j}\right\}_{j \in J}|\mu \mathrm{t} . G| \mathrm{t} \mid \text { end } \\
& T::=\mathrm{p} ?\left\{a_{i} \cdot T_{i}\right\}_{i \in I}\left|\mathrm{p} !\left\{a_{i} \cdot T_{i}\right\}_{i \in I}\right| \mu \mathrm{t} . T|\mathrm{t}| \text { end }
\end{aligned}
$$

$a_{j} \in \mathbb{A}$ corresponds to the usual message label in session type theory. We omit the mention of the carried types from the syntax in this paper, as we are not directly concerned by typing processes. Global branching type $\mathrm{p} \rightarrow \mathrm{p}^{\prime}:\left\{a_{j} \cdot G_{j}\right\}_{j \in J}$ states that participant $\mathrm{p}$ can send a message with one of the $a_{i}$ labels to participant $\mathrm{p}^{\prime}$ and that interactions described in $G_{j}$ follow. We require $\mathrm{p} \neq \mathrm{p}^{\prime}$ to prevent self-sent messages. Recursive type $\mu \mathrm{t} . G$ is for recursive protocols, assuming that type variables $\left(\mathrm{t}, \mathrm{t}^{\prime}, \ldots\right)$ are guarded in the standard way, i.e. they only occur under branchings. Type end represents session termination (often omitted). $\mathrm{p} \in G$ means that $\mathrm{p}$ appears in $G$.

Concerning local types, the branching type $\mathrm{p} ?\left\{a_{i} . T_{i}\right\}_{i \in I}$ specifies the reception of a message from $\mathrm{p}$ with a label among the $a_{i}$. The selection type $\mathrm{p} !\left\{a_{i} \cdot T_{i}\right\}_{i \in I}$ is its dual. The remaining type constructors are the same as global types. When branching is a singleton, we write $\mathrm{p} \rightarrow \mathrm{p}^{\prime}: a . G^{\prime}$ for global, and $\mathrm{p} ! a . T$ or $\mathrm{p} ? a . T$ for local.

Projection The relation between global and local types is formalised by projection. Instead of the restricted original projection [3], we use the extension with the merging operator $\bowtie$ from [10]: it allows each branch of the global type to actually contain different interaction patterns.

Definition 3.1 (projection). The projection of $G$ onto $\mathrm{p}$ (written $G\lceil\mathrm{p}$ ) is defined as:

\footnotetext{
${ }^{3}$ The terminology follows [7].
} 


$$
\begin{aligned}
& \mathrm{p} \rightarrow \mathrm{p}^{\prime}:\left\{a_{j} \cdot G_{j}\right\}_{j \in J}\left\lceil\mathrm{q}= \begin{cases}\mathrm{p} !\left\{a_{j} \cdot G_{j}\lceil\mathrm{q}\}_{j \in J}\right. & \mathrm{q}=\mathrm{p} \\
\mathrm{p} ?\left\{a_{j} \cdot G_{j}\lceil\mathrm{q}\}_{j \in J}\right. & \mathrm{q}=\mathrm{p}^{\prime} \\
\sqcup_{j \in J} G_{j}\lceil\mathrm{q} & \text { otherwise }\end{cases} \right. \\
& (\mu \mathrm{t} . G)\left\lceil\mathrm{p}=\left\{\begin{array}{ll}
\mu \mathrm{t} . G\lceil\mathrm{p} & G\lceil\mathrm{p} \neq \mathrm{t} \\
\text { end } & \text { otherwise }
\end{array} \quad \mathrm{t} \uparrow \mathrm{p}=\mathrm{t} \quad \text { end } \uparrow \mathrm{p}=\right.\text { end }\right.
\end{aligned}
$$

The mergeability relation $\bowtie$ is the smallest congruence relation over local types such that:

$$
\frac{\forall i \in(K \cap J) . T_{i} \bowtie T_{i}^{\prime} \quad \forall k \in(K \backslash J), \forall j \in(J \backslash K) \cdot a_{k} \neq a_{j}}{\mathrm{p} ?\left\{a_{k} \cdot T_{k}\right\}_{k \in K} \bowtie \mathrm{p} ?\left\{a_{j} \cdot T_{j}^{\prime}\right\}_{j \in J}}
$$

When $T_{1} \bowtie T_{2}$ holds, we define the operation $\sqcup$ as a partial commutative operator over two types such that $T \sqcup T=T$ for all types and that:

$\mathrm{p} ?\left\{a_{k} \cdot T_{k}\right\}_{k \in K} \sqcup \mathrm{p} ?\left\{a_{j} \cdot T_{j}^{\prime}\right\}_{j \in J}=\mathrm{p} ?\left(\left\{a_{k} \cdot\left(T_{k} \sqcup T_{k}^{\prime}\right)\right\}_{k \in K \cap J} \cup\left\{a_{k} \cdot T_{k}\right\}_{k \in K \backslash J} \cup\left\{a_{j} \cdot T_{j}^{\prime}\right\}_{j \in J \backslash K}\right)$

and homomorphic for other types (i.e. $\mathscr{C}\left[T_{1}\right] \sqcup \mathscr{C}\left[T_{2}\right]=\mathscr{C}\left[T_{1} \sqcup T_{2}\right]$ where $\mathscr{C}$ is a context for local types). We say that $G$ is well-formed if for all $\mathrm{p} \in \mathcal{P}, G\lceil\mathrm{p}$ is defined.

Example 3.1 (Commit). The global type for the commit protocol in Figure 1 is:

$\mu$ t.A $\rightarrow$ B $:\{$ act. $\mathrm{B} \rightarrow \mathrm{C}:\{$ sig. $\mathrm{A} \rightarrow \mathrm{C}:$ commit.t $\}$, quit. $\mathrm{B} \rightarrow \mathrm{C}:\{$ save $. \mathrm{A} \rightarrow \mathrm{C}:$ finish.end $\}\}$

Then C's local type is: $\mu$ t.B? sig.A?\{commit.t\}, save.A?\{finish.end\}\}.

LTS over global types We next present new labelled transition relations (LTS) for global and local types and their sound and complete correspondence.

The first step for giving a LTS semantics to global types (and then to local types) is to designate the observables $\left(\ell, \ell^{\prime}, \ldots\right)$. We choose here to follow the definition of actions for CFSMs where a label $\ell$ denotes the sending or the reception of a message of label $a$ from $\mathrm{p}$ to $\mathrm{p}^{\prime}: \ell::=\mathrm{pp}^{\prime} ! a \mid \mathrm{pp}^{\prime} ? a$

In order to define an LTS for global types, we need to represent intermediate states in the execution. For this reason, we introduce in the grammar of $G$ the construct $\mathrm{p} \rightsquigarrow$ $\mathrm{p}^{\prime}: a_{j} \cdot G_{j}$ to represent the fact that the message $a_{j}$ has been sent but not yet received.

Definition 3.2 (LTS over global types). The relation $G \stackrel{\ell}{\rightarrow} G^{\prime}$ is defined as $(\operatorname{subj}(\ell)$ is defined in $\S 2$ ):

$$
\begin{gathered}
{[\mathrm{GR} 1] \quad \mathrm{p} \rightarrow \mathrm{p}^{\prime}:\left\{a_{i} \cdot G_{i}\right\}_{i \in I} \stackrel{\mathrm{pp}^{\prime} ! a_{j}}{\longrightarrow} \mathrm{p} \rightsquigarrow \mathrm{p}^{\prime}: j\left\{a_{i} \cdot G_{i}\right\}_{i \in I} \quad(j \in I)} \\
{[\mathrm{GR} 2] \quad \mathrm{p} \rightsquigarrow \mathrm{p}^{\prime}: j\left\{a_{i} \cdot G_{i}\right\}_{i \in I} \stackrel{\mathrm{pp}^{\prime} ? a_{j}}{\longrightarrow} G_{j} \quad[\mathrm{GR} 3] \frac{G[\mu \mathrm{t} . G / \mathrm{t}] \stackrel{\ell}{\rightarrow} G^{\prime}}{\mu \mathrm{t} \cdot G \stackrel{\ell}{\rightarrow} G^{\prime}}} \\
{[\mathrm{GR} 4] \frac{\forall j \in I \quad G_{j} \stackrel{\ell}{\rightarrow} G_{j}^{\prime} \quad \mathrm{p}, \mathrm{q} \notin \operatorname{subj}(\ell)}{\mathrm{p} \rightarrow \mathrm{q}:\left\{a_{i} \cdot G_{i}\right\}_{i \in I} \stackrel{\ell}{\rightarrow} \mathrm{p} \rightarrow \mathrm{q}:\left\{a_{i} \cdot G_{i}^{\prime}\right\}_{i \in I}}[\mathrm{GR} 5] \frac{G_{j} \stackrel{\ell}{\rightarrow} G_{j}^{\prime} \quad \mathrm{q} \notin \operatorname{subj}(\ell) \quad \forall i \in I \backslash j, G_{i}^{\prime}=G_{i}}{\mathrm{p} \rightsquigarrow \mathrm{q}: j\left\{a_{i} \cdot G_{i}\right\}_{i \in I} \stackrel{\ell}{\rightarrow} \mathrm{p} \rightsquigarrow \mathrm{q}: j\left\{a_{i} \cdot G_{i}^{\prime}\right\}_{i \in I}}}
\end{gathered}
$$

[GR1] represents the emission of a message while [GR2] describes the reception of a message. [GR3] governs recursive types. [GR4,5] define the asynchronous semantics of global types, where the syntactic order of messages is enforced only for the participants that are involved. For example, in the case when the participants of two consecutive communications are disjoint, as in: $G_{1}=\mathrm{A} \rightarrow \mathrm{B}: a . \mathrm{C} \rightarrow \mathrm{D}: b$.end, we can observe 
the emission (and possibly the reception) of $b$ before the emission (or reception) of $a$ (by [GR4]).

A more interesting example is: $G_{2}=\mathrm{A} \rightarrow \mathrm{B}: a . \mathrm{A} \rightarrow \mathrm{C}: b$.end. We write $\ell_{1}=A B ! a$, $\ell_{2}=A B ? a, \ell_{3}=A C ! b$ and $\ell_{4}=A C ? b$. The LTS allows the following three sequences:

$$
\begin{aligned}
& G_{1} \stackrel{\ell_{1}}{\rightarrow} \mathrm{A} \rightsquigarrow \mathrm{B}: a . \mathrm{A} \rightarrow \mathrm{C}: b . \text { end } \stackrel{\ell_{2}}{\rightarrow} \mathrm{A} \rightarrow \mathrm{C}: b \text {.end } \quad \stackrel{\ell_{3}}{\rightarrow} \mathrm{A} \rightsquigarrow \mathrm{C}: b \text {.end } \stackrel{\ell_{4}}{\rightarrow} \text { end } \\
& G_{1} \stackrel{\ell_{1}}{\rightarrow} \mathrm{A} \rightsquigarrow \mathrm{B}: a . \mathrm{A} \rightarrow \mathrm{C}: b \text {.end } \stackrel{\ell_{3}}{\rightarrow} \mathrm{A} \rightsquigarrow \mathrm{B}: a \cdot \mathrm{A} \rightsquigarrow \mathrm{C}: b \text {.end } \stackrel{\ell_{2}}{\rightarrow} \mathrm{A} \rightsquigarrow \mathrm{C}: b \text {.end } \stackrel{\ell_{4}}{\rightarrow} \text { end } \\
& G_{1} \stackrel{\ell_{1}}{\longrightarrow} \mathrm{A} \rightsquigarrow \mathrm{B}: a \cdot \mathrm{A} \rightarrow \mathrm{C}: b \text {.end } \stackrel{\ell_{3}}{\longrightarrow} \mathrm{A} \rightsquigarrow \mathrm{B}: a \cdot \mathrm{A} \rightsquigarrow \mathrm{C}: b \text {.end } \stackrel{\ell_{4}}{\longrightarrow} \mathrm{A} \rightsquigarrow \mathrm{B}: a \text {.end } \stackrel{\ell_{2}}{\longrightarrow} \text { end }
\end{aligned}
$$

The last sequence is the most interesting: the sender $A$ has to follow the syntactic order but the receiver $C$ can get the message $b$ before $B$ receives $a$. The respect of these constraints is enforced by the conditions $\mathrm{p}, \mathrm{q} \notin \operatorname{subj}(\ell)$ and $\mathrm{q} \notin \operatorname{subj}(\ell)$ in rules [GR4,5].

LTS over local types We define the LTS over local types. This is done in two steps, following the model of CFSMs, where the semantics is given first for individual automata and then extended to communicating systems. We use the same labels $\left(\ell, \ell^{\prime}, \ldots\right)$ as the ones for CFSMs.

Definition 3.3 (LTS over local types). The relation $T \stackrel{\ell}{\rightarrow} T^{\prime}$, for the local type of role $\mathrm{p}$, is defined as:

$$
[\mathrm{LR} 1] \mathrm{q} !\left\{a_{i} \cdot T_{i}\right\}_{i \in I} \stackrel{\mathrm{pq} ! a_{i}}{\longrightarrow} T_{i} \quad[\mathrm{LR} 2] \mathrm{q} ?\left\{a_{i} \cdot T_{i}\right\}_{i \in I} \stackrel{\mathrm{qp} ? a_{j}}{\longrightarrow} T_{j} \quad[\mathrm{LR} 3] \frac{T[\mu \mathrm{t} . T / \mathrm{t}] \stackrel{\ell}{\rightarrow} T^{\prime}}{\mu \mathrm{t} . T \stackrel{\ell}{\rightarrow} T^{\prime}}
$$

The semantics of a local type follows the intuition that every action of the local type should obey the syntactic order. We define the LTS for collections of local types.

Definition 3.4 (LTS over collections of local types). A configuration $s=(\vec{T} ; \vec{w})$ of a system of local types $\left\{T_{\mathrm{p}}\right\}_{\mathrm{p} \in \mathcal{P}}$ is a pair with $\vec{T}=\left(T_{\mathrm{p}}\right)_{\mathrm{p} \in \mathcal{P}}$ and $\vec{w}=\left(w_{\mathrm{pq}}\right)_{\mathrm{p} \neq \mathrm{q} \in \mathcal{P}}$ with $w_{\mathrm{pq}} \in \mathbb{A}^{*}$. We then define the transition system for configurations. For a configuration $s_{T}=(\vec{T} ; \vec{w})$, the visible transitions of $s_{T} \stackrel{\ell}{\rightarrow} s_{T}^{\prime}=\left(\vec{T}^{\prime} ; \vec{w}^{\prime}\right)$ are defined as:

1. $T_{\mathrm{p}} \stackrel{\mathrm{pq} ! a}{\longrightarrow} T_{\mathrm{p}}^{\prime}$ and (a) $T_{\mathrm{p}^{\prime}}^{\prime}=T_{\mathrm{p}^{\prime}}$ for all $\mathrm{p}^{\prime} \neq \mathrm{p}$; and (b) $w_{\mathrm{pq}}^{\prime}=w_{\mathrm{pq}} \cdot a$ and $w_{\mathrm{p}^{\prime} \mathrm{q}^{\prime}}^{\prime}=w_{\mathrm{p}^{\prime} \mathrm{q}^{\prime}}$ for all $\mathrm{p}^{\prime} \mathrm{q}^{\prime} \neq \mathrm{pq}$; or

2. $T_{\mathrm{q}} \stackrel{\mathrm{pq} ? a}{\longrightarrow} T_{\mathrm{q}}^{\prime}$ and (a) $T_{\mathrm{p}^{\prime}}^{\prime}=T_{\mathrm{p}^{\prime}}$ for all $\mathrm{p}^{\prime} \neq \mathrm{q}$; and (b) $w_{\mathrm{pq}}=a \cdot w_{\mathrm{pq}}^{\prime}$ and $w_{\mathrm{p}^{\prime} \mathrm{q}^{\prime}}^{\prime}=w_{\mathrm{p}^{\prime} \mathrm{q}^{\prime}}$ for all $\mathrm{p}^{\prime} \mathrm{q}^{\prime} \neq \mathrm{pq}$.

The semantics of local types is therefore defined over configurations, following the definition of the semantics of CFSMs. $w_{\text {pq }}$ represents the FIFO queue at channel pq. We write $\operatorname{Tr}(G)$ to denote the set of the visible traces that can be obtained by reducing $G$. Similarly for $\operatorname{Tr}(T)$ and $\operatorname{Tr}(S)$. We extend the trace equivalences $\approx$ and $\approx_{n}$ in $\S 2$ to global types and configurations of local types.

We now state the soundness and completeness of projection with respect to the LTSs defined above. The proof is given in Appendix A.1.

Theorem 3.1 (soundness and completeness). ${ }^{4}$ Let $G$ be a global type with participants $\mathcal{P}$ and let $\vec{T}=\left\{G\lceil\mathrm{p}\}_{\mathrm{p} \in \mathcal{P}}\right.$ be the local types projected from $G$. Then $G \approx(\vec{T} ; \vec{\varepsilon})$.

\footnotetext{
${ }^{4}$ The local type abstracts the behaviour of multiparty typed processes as proved in the subject reduction theorem in [17]. Hence this theorem implies that processes typed by global type $G$ by the typing system in $[3,17]$ follow the LTS of $G$.
} 
Local types and CFSMs Next we show how to algorithmically go from local types to CFSMs and back while preserving the trace semantics. We start by translating local types into CFSMs.

Definition 3.5 (translation from local types to CFSMs). Write $T^{\prime} \in T$ if $T^{\prime}$ occurs in $T$. Let $T_{0}$ be the local type of participant p projected from $G$. The automaton corresponding to $T_{0}$ is $\mathcal{A}\left(T_{0}\right)=\left(Q, C, q_{0}, \mathbb{A}, \delta\right)$ where: (1) $Q=\left\{T^{\prime} \mid T^{\prime} \in T_{0}, T^{\prime} \neq \mathrm{t}, T^{\prime} \neq \mu \mathrm{t} . T\right\} ;(2)$ $q_{0}=T_{0}^{\prime}$ with $T_{0}=\mu \overrightarrow{\mathrm{t}} . T_{0}^{\prime}$ and $T_{0}^{\prime} \in Q ;(3) C=\{\mathrm{pq} \mid \mathrm{p}, \mathrm{q} \in G\} ;(4) \mathbb{A}$ is the set of $\{a \in G\}$; and (5) $\delta$ is defined as:

$$
\begin{aligned}
& \text { If } T=\mathrm{p}^{\prime} !\left\{a_{j} . T_{j}\right\}_{j \in J} \in Q \text {, then } \begin{cases}\left(T,\left(\mathrm{pp}^{\prime} ! a_{j}\right), T_{j}\right) \in \delta & T_{j} \neq \mathrm{t} \\
\left(T,\left(\mathrm{pp}^{\prime} ! a_{j}\right), T^{\prime}\right) \in \delta & T_{j}=\mathrm{t}, \quad \mu \mathrm{t} \overrightarrow{\mathrm{t}} . T^{\prime} \in T_{0}, T^{\prime} \in Q\end{cases} \\
& \text { If } T=\mathrm{p}^{\prime} ?\left\{a_{j} . T_{j}\right\}_{j \in J} \in Q \text {, then } \begin{cases}\left(T,\left(\mathrm{p}^{\prime} \mathrm{p} ? a_{j}\right), T_{j}\right) \in \delta & T_{j} \neq \mathrm{t} \\
\left(T,\left(\mathrm{p}^{\prime} \mathrm{p} ? a_{j}\right), T^{\prime}\right) \in \delta & T_{j}=\mathrm{t}, \mu \mathrm{tt} . T^{\prime} \in T_{0}, T^{\prime} \in Q\end{cases}
\end{aligned}
$$

The definition says that the set of states $Q$ are the suboccurrences of branching or selection or end in the local type; the initial state $q_{0}$ is the occurrence of (the recursion body of) $T_{0}$; the channels and alphabets correspond to those in $T_{0}$; and the transition is defined from the state $T$ to its body $T_{j}$ with the action $\mathrm{pp}^{\prime} ! a_{j}$ for the output and $\mathrm{pp}^{\prime} ? a_{j}$ for the input. If $T_{j}$ is a recursive type variable $t$, it points the state of the body of the corresponding recursive type. As an example of the translation, see C's local type in Example 3.1 and its corresponding automaton in Figure 1.

Proposition 3.1 (local types to CFSMs). Assume $T_{\mathrm{p}}$ is a local type. Then $\mathcal{A}\left(T_{\mathrm{p}}\right)$ is deterministic, directed and has no mixed states.

We say that a CFSM is basic if it is deterministic, directed and has no mixed states. Any basic CFSM can be translated into a local type.

Definition 3.6 (translation from a basic CFSM to a local type). $L e t M_{\mathrm{p}}=\left(Q, C, q_{0}, \mathbb{A}, \boldsymbol{\delta}\right)$ and assume $M_{\mathrm{q}}$ is basic. Then we define the translation $\mathcal{T}\left(M_{\mathrm{p}}\right)$ such that $\mathcal{T}\left(M_{\mathrm{p}}\right)=\mathcal{T}_{\mathcal{E}}\left(q_{0}\right)$ where $\mathcal{T}_{\tilde{q}}(q)$ is defined as:

(1) $\mathcal{T}_{\tilde{q}}(q)=\mu \mathrm{t}_{q} \cdot \mathrm{p}^{\prime} !\left\{a_{j} \cdot \mathcal{T}_{\tilde{q} \cdot q}^{\circ}\left(q_{j}\right)\right\}_{j \in J}$ if $\left(q, \mathrm{pp}^{\prime} ! a_{j}, q_{j}\right) \in \delta$;

(2) $\mathcal{T}_{\tilde{q}}(q)=\mu \mathrm{t}_{q} \cdot \mathrm{p}^{\prime} ?\left\{a_{j} \cdot \mathcal{T}_{\tilde{q} \cdot q}^{\circ}\left(q_{j}\right)\right\}_{j \in J}$ if $\left(q, \mathrm{p}^{\prime} \mathrm{p} ? a_{j}, q_{j}\right) \in \delta$;

(3) $\mathcal{T}_{\tilde{q}}^{\circ}(q)=\mathcal{T}_{\mathcal{E}}(q)=$ end if $q$ is final; (4) $\mathcal{T}_{\tilde{q}}^{\circ}(q)=\mathrm{t}_{q_{k}}$ if $\left(q, \ell, q_{k}\right) \in \delta$ and $q_{k} \in \tilde{q}$; and

(5) $\mathcal{T}_{\tilde{q}}^{\circ}(q)=\mathcal{T}_{\tilde{q}}(q)$ otherwise.

Finally, we replace $\mu \mathrm{t} . T$ by $T$ if $\mathrm{t}$ is not in $T$.

In $\mathcal{T}_{\tilde{q}}, \tilde{q}$ records visited states; $(1,2)$ translate the receiving and sending states to branching and selection types, respectively; (3) translates the final state to end; and (4) is the case of a recursion: since $q_{k}$ was visited, $\ell$ is dropped and replaced by the type variable.

The following states that the translations preserve the semantics.

Proposition 3.2 (translations between CFSMs and local types). If a CFSM $M$ is basic, then $M \approx \mathcal{T}(M)$. If $T$ is a local type, then $T \approx \mathcal{A}(T)$. 


\section{Completeness and synthesis}

This section studies the synthesis and sound and complete characterisation of the multiparty session types as communicating automata. We first note that basic CFSMs correspond to the natural generalisation of half-duplex systems $[8, \S 4.1 .1]$, in which each pair of machines linked by two channels, one in each direction, communicates in a half-duplex way. In this class, the safety properties of Definition 2.4 are however undecidable [8, Theorem 36]. We therefore need a stronger (and decidable) property to force basic CFSMs to behave as if they were the result of a projection from global types.

Multiparty compatibility In the two machines case, there exists a sound and complete condition called compatible [15]. Let us define the isomorphism $\Phi:(C \times\{!, ?\} \times$ $\mathbb{A})^{*} \longrightarrow(C \times\{!, ?\} \times \mathbb{A})^{*}$ such that $\Phi(j ? a)=j ! a, \Phi(j ! a)=j ? a, \Phi(\varepsilon)=\varepsilon, \Phi\left(t_{1} \cdots t_{n}\right)=$ $\Phi\left(t_{1}\right) \cdots \Phi\left(t_{n}\right)$. $\Phi$ exchanges a sending action with the corresponding receiving one and vice versa. The compatibility of two machines can be immediately defined as $\operatorname{Tr}\left(M_{1}\right)=\Phi\left(\operatorname{Tr}\left(M_{2}\right)\right)$ (i.e. the traces of $M_{1}$ are exactly the set of dual traces of $M_{2}$ ). The idea of the extension to the multiparty case comes from the observation that from the viewpoint of the participant $\mathrm{p}$, the rest of all the machines $\left(M_{\mathrm{q}}\right)_{\mathrm{q} \in \mathcal{P} \backslash \mathrm{p}}$ should behave as if they were one CFSM which offers compatible traces $\Phi\left(\operatorname{Tr}\left(M_{\mathrm{p}}\right)\right)$, up to internal synchronisations (i.e. 1-bounded executions). Below we define a way to group CFSMs.

Definition 4.1 (Definition 37, [8]). Let $M_{i}=\left(Q_{i}, C_{i}, q_{0 i}, \mathbb{A}_{i}, \delta_{i}\right)$. The associated CFSM of $S=\left(M_{1}, . ., M_{n}\right)$ is $M=\left(Q, C, q_{0}, \Sigma, \delta\right)$ such that: $Q=Q_{1} \times Q_{2} \times \cdots \times Q_{n}, q_{0}=$ $\left(q_{01}, \ldots, q_{0 n}\right)$ and $\delta$ is the least relation verifying: $\left(\left(q_{1}, \ldots, q_{i}, \ldots, q_{n}\right), \ell,\left(q_{1}, \ldots, q_{i}^{\prime}, \ldots, q_{n}\right)\right) \in$ $\delta$ if $\left(q_{i}, \ell, q_{i}^{\prime}\right) \in \delta_{i}(1 \leq i \leq n)$.

Below we define a notion of compatibility extended to more than two CFSMs. We say that $\varphi$ is an alternation if $\varphi$ is an alternation of sending and corresponding receive actions (i.e. the action pq! $a$ is immediately followed by pq?a).

Definition 4.2 (multiparty compatible system). A system $S=\left(M_{1}, \ldots, M_{n}\right)(n \geq 2)$ is multiparty compatible if for any 1-bounded reachable stable state $s \in R S_{1}(S)$, for any sequence of actions $\ell_{1} \cdots \ell_{k}$ from $s$ in $M_{i}$, there is a sequence of transitions $\varphi_{1} \cdot t_{1} \cdot \varphi_{2} \cdot t_{2}$. $\varphi_{3} \cdots \varphi_{k} \cdot t_{k}$ from $s$ in a CFSM corresponding to $S^{-i}=\left(M_{1}, . ., M_{i-1}, M_{i+1}, . ., M_{n}\right)$ where $\varphi_{j}$ is either empty or an alternation, $\ell_{j}=\Phi\left(\operatorname{act}\left(t_{j}\right)\right)$ and $i \notin \operatorname{act}\left(\varphi_{j}\right)$ for $1 \leq j \leq k$ (i.e. $\varphi_{j}$ does not contain actions to or from channel $i$ ).

The above definition states that for each $M_{i}$, the rest of machines $S^{-i}$ can produce the compatible (dual) actions by executing alternations in $S^{-i}$. From $M_{i}$, these intermediate alternations can be seen as non-observable internal actions.

Example 4.1 (multiparty compatibility). As an example, we can test the multiparty compatibility property on the commit example of Figure 1. We only detail here how to check the compatibility from the point of view of $\mathrm{C}$. To check the compatibility for the actions $\operatorname{act}\left(t_{1} \cdot t_{2}\right)=B C$ ? $\mathrm{sig} \cdot A C$ ! commit, the only possible 1-bound (i.e. alternating) execution is $A B$ !act $\cdot A B$ ?act, and $\Phi\left(\operatorname{act}\left(t_{1}\right)\right)=B C$ !sig sent from B and $\Phi\left(\operatorname{act}\left(t_{2}\right)\right)=$ $A C$ ! commit sent from $A$. To check the compatibility for the actions $\operatorname{act}\left(t_{3} \cdot t_{4}\right)=B C$ ? save . $A C$ ?finish, the 1-bound execution is $A B$ ! quit $\cdot A B$ ?quit, and $\Phi\left(\operatorname{act}\left(t_{3}\right)\right)=B C$ ! save from $\mathrm{B}$ and $\Phi\left(\operatorname{act}\left(t_{4}\right)\right)=A C$ !finish from $\mathrm{A}$. 
Remark 4.1. In Definition 4.2, we require to check the compatibility from any 1-bounded reachable stable state in the case one branch is selected by different senders. Consider the following machines:
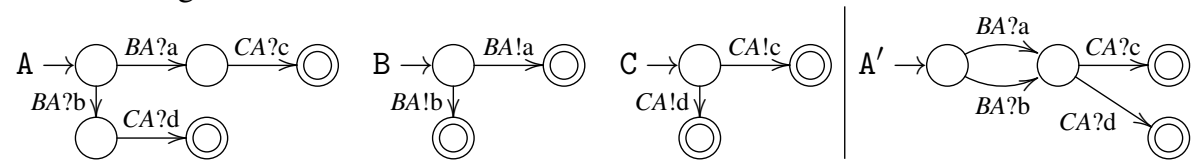

In $A, B$ and $C$, each action in each machine has its dual but they do not satisfy multiparty compatibility. For example, if $B A ! \mathrm{a} \cdot B A$ ? a is executed, $C A$ !d does not have a dual action (hence they do not satisfy the safety properties). On the other hand, the machines $A^{\prime}, B$ and $\mathrm{C}$ satisfy the multiparty compatibility.

Theorem 4.1. Assume $S=\left(M_{\mathrm{p}}\right)_{\mathrm{p} \in \mathcal{P}}$ is basic and multiparty compatible. Then $S$ satisfies the three safety properties in Definition 2.4. Further, if there exists at least one $M_{\mathrm{q}}$ which includes a final state, then $S$ satisfies the liveness property.

Proof. We first prove that any basic $S$ which satisfies multiparty compatible is stable ( $S$ is stable, if, for all $s \in R S(S)$, there exists an execution $\stackrel{\varphi^{\prime}}{\rightarrow}$ such that $s \stackrel{\varphi^{\prime}}{\rightarrow} s^{\prime}$ and $s^{\prime}$ is stable, and there is a 1-bounded execution $s_{0} \stackrel{\varphi^{\prime \prime}}{\rightarrow} s^{\prime}$, i.e. any trace can be translated into a 1-bounded execution after some appropriate executions). The proof is non-trivial using a detailed analysis of causal relations to translate into a 1-bounded executions. Then the orphan message- and the reception error-freedom are its corollary. The deadlockfreedom is proved by the stable property and multiparty compatibility. Liveness is a consequence of the orphan message- and deadlock-freedom. See Appendix B.

Proposition 4.1. If all the CFSMs $M_{\mathrm{p}}(\mathrm{p} \in \mathcal{P})$ are basic, there is an algorithm to check whether $\left(M_{\mathrm{p}}\right)_{\mathrm{p} \in \mathcal{P}}$ is multiparty compatible.

Proof. The algorithm to check $M_{\mathrm{p}}$ 's compatibility with $S^{-\mathrm{p}}$ is defined using the set $R S_{1}(S)$ of reachable states using 1-bounded executions. Note that the set $R S_{1}(S)$ is decidable [8, Remark 19]. We start from $q=q_{0}$ and the initial configuration $s=s_{0}$. Suppose that, from $q$, we have the transitions $t_{i}=\left(q, \mathrm{qp} ! a_{i}, q_{i}^{\prime}\right) \in \delta_{\mathrm{p}}$. We then construct $R S_{1}(S)$ (without executing p) until it includes $s^{\prime}$ such that $\left\{s^{\prime} \stackrel{t_{i}}{\rightarrow} \stackrel{t}{\prime}_{>}^{\prime} s_{j}\right\}_{j \in J}$ where $\operatorname{act}\left(t_{i}^{\prime}\right)=$ $\mathrm{qp} ? a_{i}$ and $I \subseteq J$. If there exists no such $s^{\prime}$, it returns false and terminates. The case where, from $q$, we have receiving transitions $t=\left(q, \mathrm{qp} ? a_{i}, q_{i}^{\prime}\right)$ is dual. If it does not fail, we continue to check from state $q_{i}^{\prime}$ and configuration $s_{i}$ for each $i \in I$. We repeat this procedure until we visit all $q \in Q_{\mathrm{p}}$. Then repeat for the other machines $\mathrm{p}^{\prime}$ such that $\mathrm{p}^{\prime} \in \mathcal{P} \backslash \mathrm{p}$. Then we repeat this procedure for all stable $s \in R S_{1}(S)$.

Synthesis Below we state the lemma which will be crucial for the proof of the synthesis and completeness. The lemma comes from the intuition that the transitions of multiparty compatible systems are always permutations of one-bounded executions as it is the case in multiparty session types. See Appendix B.2 for the proof.

Lemma 4.1 (1-buffer equivalence). Suppose $S_{1}$ and $S_{2}$ are two basic and multiparty compatible communicating systems such that $S_{1} \approx_{1} S_{2}$, then $S_{1} \approx S_{2}$.

Theorem 4.2 (synthesis). Suppose $S$ is a basic system and multiparty compatible. Then there is an algorithm which successfully builds well-formed $G$ such that $S \approx G$ if such $G$ exists, and otherwise terminates. 


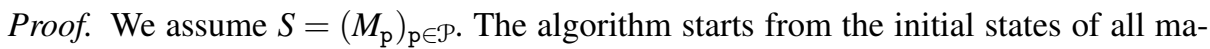
chines $\left(q^{\mathrm{p}_{1}} 0, \ldots, q^{\mathrm{p}_{n}} 0\right)$. We take a pair of the initial states which is a sending state $q_{0}^{\mathrm{p}}$ and a receiving state $q_{0}^{\mathrm{q}}$ from $\mathrm{p}$ to $\mathrm{q}$. We note that by directness, if there are more than two pairs, the participants in two pairs are disjoint, and by [G4] in Definition 3.2, the order does not matter. We apply the algorithm with the invariant that all buffers are empty and that we repeatedly pick up one pair such that $q_{\mathrm{p}}$ (sending state) and $q_{\mathrm{q}}$ (receiving state). We define $G\left(q_{1}, \ldots, q_{n}\right)$ where $\left(q_{\mathrm{p}}, q_{\mathrm{q}} \in\left\{q_{1}, \ldots, q_{n}\right\}\right)$ as follows:

- if $\left(q_{1}, \ldots, q_{n}\right)$ has already been examined and if all participants have been involved since then (or the ones that have not are in their final state), we set $G\left(q_{1}, \ldots, q_{n}\right)$ to be $t_{q_{1}, \ldots, q_{n}}$. Otherwise, we select a pair sender/receiver from two participants that have not been involved (and are not final) and go to the next step;

- otherwise, in $q_{\mathrm{p}}$, from machine $\mathrm{p}$, we know that all the transitions are sending actions towards $\mathrm{p}^{\prime}$ (by directedness), i.e. of the form $\left(q_{\mathrm{p}}, \mathrm{pq} ! a_{i}, q_{i}\right) \in \delta_{\mathrm{p}}$ for $i \in I$.

- we check that machine $\mathrm{q}$ is in a receiving state $q_{\mathrm{q}}$ such that $\left(q_{\mathrm{q}}, \mathrm{pq} ? a_{j}, q_{j}^{\prime}\right) \in \delta_{\mathrm{p}^{\prime}}$ with $j \in J$ and $I \subseteq J$.

- we set $\mu \mathrm{t}_{q_{1}, \ldots, q_{n}} \cdot \mathrm{p} \rightarrow \mathrm{q}:\left\{a_{i} \cdot G\left(q_{1}, \ldots, q_{\mathrm{p}} \leftarrow q_{i}, \ldots, q_{\mathrm{q}} \leftarrow q_{i}^{\prime}, \ldots, q_{n}\right)\right\}_{i \in I}$ (we replace $q_{\mathrm{p}}$ and $q_{\mathrm{q}}$ by $q_{i}$ and $q_{i}^{\prime}$, respectively) and continue by recursive calls.

- if all sending states in $q_{1}, \ldots, q_{n}$ become final, then we set $G\left(q_{1}, \ldots, q_{n}\right)=$ end.

- we erase unnecessary $\mu$ t if $\mathrm{t} \notin G$ and check $G$ satisfies Definition 3.1.

Since the algorithm only explores 1-bounded executions, the reconstructed $G$ satisfies $G \approx_{1} S$. By Theorem 3.1, we know that $G \approx\left(\left\{G\lceil\mathrm{p}\}_{\mathrm{p} \in \mathcal{P}} ; \vec{\varepsilon}\right)\right.$. Hence, by Proposition 3.2, we have $G \approx S^{\prime}$ where $S^{\prime}$ is the communicating system translated from the projected local types $\left\{G\lceil\mathrm{p}\}_{\mathrm{p} \in \mathcal{P}}\right.$ of $G$. By Lemma $4.1, S \approx S^{\prime}$ and therefore $S \approx G$.

The algorithm can generate the global type in Example 3.1 from CFSMs in Figure 1 and the global type $\mathrm{B} \rightarrow \mathrm{A}\{a: \mathrm{C} \rightarrow \mathrm{A}:\{c:$ end, $d:$ end $\}, b: \mathrm{C} \rightarrow \mathrm{A}:\{c:$ end, $d:$ end $\}\}$ from $\mathrm{A}^{\prime}, \mathrm{B}$ and $\mathrm{C}$ in Remark 4.1. Note that $\mathrm{B} \rightarrow \mathrm{A}\{a: \mathrm{C} \rightarrow \mathrm{A}:\{c:$ end $\}, b: \mathrm{C} \rightarrow \mathrm{A}:\{d:$ end $\}\}$ generated by A, B and C in Remark 4.1 is not projectable by Definition 3.1, hence it is not well-formed.

By Theorems 3.1, 4.1 and 4.2, and Proposition 3.2, we can now conclude:

Theorem 4.3 (soundness and completeness in CMSA). Suppose $S$ is basic and multiparty compatible. Then there exists $G$ such that $S \approx G$. Conversely, if $G$ is well-formed, then there exists $S$ which satisfies the three safety properties in Definition 2.4 and $S \approx G$.

\section{Conclusion and related work}

This paper investigated the sound and complete characterisation of multiparty session types into CFSMs and developed a decidable synthesis algorithm from basic CFSMs. The main tool we used is a new extension to multiparty interactions of the duality condition for binary session types, called multiparty compatibility. The basic condition (coming from the binary session types) and the multiparty compatibility property are a necessary and sufficient condition to obtain safe global types. Our aim is to offer a duality notion which would be applicable to extend other theoretical foundations such as the Curry-Howard correspondence with linear logics $[6,26]$ to multiparty communications. Basic multiparty compatible CFSMs also define one of the few non-trivial decidable subclass of CFSMs which satisfy deadlock-freedom. The methods proposed here are palatable to a wide range of applications based on choreography protocol models and 
more widely, finite state machines. We are currently working on two applications based on the theory developed in this paper: the Testable Architecture [23] which enables the communication structure of the implementation to be inferred and to be tested against the choreography; and dynamic monitoring for a large scale cyberinfrastructure in [22] where a central controller can check that distributed update paths for monitor specifications (which form FSMs projected from a global specification) are safe by synthesis.

Our previous work [11] presented the first translation from global and local types into CFSMs. It only analysed the properties of the automata resulting from such a translation. The complete characterisation of global types independently from the projected local types was left open, as was synthesis. This present paper closes this open problem. There are a large number of paper that can be found in the literature about the synthesis of CFSMs. See [20] for a summary of recent results. The main distinction with CFSM synthesis is, apart from the formal setting (i.e. types), about the kind of the target specifications to be generated (global types in our case). Not only our synthesis is concerned about trace properties (languages) like the standard synthesis of CFSMs (the problem of the closed synthesis of CFSMs is usually defined as the construction from a regular language $L$ of a machine satisfying certain conditions related to buffer boundedness, deadlock-freedom and words swapping), but we also generate concrete syntax or choreography descriptions as types of programs or software. Hence they are directly applicable to programming languages and can be straightforwardly integrated into the existing frameworks that are based on session types.

Within the context of multiparty session types, [19] first studied the reconstruction of a global type from its projected local types up to asynchronous subtyping and [18] recently offers a typing system to synthesise global types from local types. Our synthesis based on CFSMs is more general since CFSMs do not depend on the syntax. For example, $[18,19]$ cannot treat the synthesis for $\mathrm{A}^{\prime}$, B and C in Remark 4.1. These works also do not study the completeness (i.e. they build a global type from a set of projected local types (up to subtyping), and do not investigate necessary and sufficient conditions to build a well-formed global type). A difficulty of the completeness result is that it is generally unknown if the global type constructed by the synthesis can simulate executions with arbitrary buffer bounds since the synthesis only directly looks at 1-bounded executions. In this paper, we proved Lemma 4.1 and bridged this gap towards the complete characterisation. Recent work by [2,7] focus on proving the semantic correspondence between global and local descriptions (see [11] for more detailed comparison), but no synthesis algorithm is studied.

\section{References}

1. E. Badouel and P. Darondeau. Theory of regions. Lectures on Petri Nets I: Basic Models, pages 529-586, 1998.

2. S. Basu, T. Bultan, and M. Ouederni. Deciding choreography realizability. In POPL'12, pages 191-202. ACM, 2012.

3. L. Bettini et al. Global progress in dynamically interleaved multiparty sessions. In CONCUR, volume 5201 of $L N C S$, pages 418-433, 2008.

4. Business Process Model and Notation. http://www.bpmn. org.

5. D. Brand and P. Zafiropulo. On communicating finite-state machines. J. ACM, 30:323-342, April 1983.

6. L. Caires and F. Pfenning. Session types as intuitionistic linear propositions. In CONCUR, volume 6269 of $L N C S$, pages 222-236. Springer, 2010.

7. G. Castagna, M. Dezani-Ciancaglini, and L. Padovani. On global types and multi-party session. LMCS, 8(1), 2012. 
8. G. Cécé and A. Finkel. Verification of programs with half-duplex communication. Inf. Comput., 202(2):166-190, 2005.

9. J. Cortadella, M. Kishinevsky, L. Lavagno, and A. Yakovlev. Deriving petri nets from finite transition systems. Computers, IEEE Transactions on Computers, 47(8):859-882, 1998.

10. P.-M. Deniélou and N. Yoshida. Dynamic multirole session types. In POPL, pages 435-446. ACM, 2011. Full version, Prototype at http://www.doc.ic.ac.uk/ pmalo/dynamic.

11. P.-M. Deniélou and N. Yoshida. Multiparty session types meet communicating automata. In ESOP, volume 7211 of $L N C S$, pages 194-213. Springer, 2012.

12. M. Fähndrich, M. Aiken, C. Hawblitzel, O. Hodson, G. C. Hunt, J. R. Larus, , and S. Levi. Language Support for Fast and Reliable Message-based Communication in Singularity OS. In EuroSys2006, ACM SIGOPS, pages 177-190. ACM Press, 2006.

13. B. Genest, A. Muscholl, and D. Peled. Message sequence charts. In Lectures on Concurrency and Petri Nets, volume 3098 of LNCS, pages 537-558, 2004.

14. J.-Y. Girard. Linear logic. TCS, 50, 1987.

15. M. Gouda, E. Manning, and Y. Yu. On the progress of communication between two finite state machines. Information and Control., 63:200-216, 1984.

16. K. Honda, V. T. Vasconcelos, and M. Kubo. Language primitives and type disciplines for structured communication-based programming. In ESOP'98, volume 1381 of $L N C S$, pages 22-138. Springer, 1998.

17. K. Honda, N. Yoshida, and M. Carbone. Multiparty Asynchronous Session Types. In POPL'08, pages 273-284. ACM, 2008.

18. J. Lange and E. Tuosto. Synthesising choreographies from local session types. In CONCUR, volume 7454 of $L N C S$, pages 225-239. Springer, 2012.

19. D. Mostrous, N. Yoshida, and K. Honda. Global principal typing in partially commutative asynchronous sessions. In ESOP'09, volume 5502 of LNCS, pages 316-332. Springer, 2009.

20. A. Muscholl. Analysis of communicating automata. In LATA, volume 6031 of LNCS, pages 50-57. Springer, 2010.

21. M. Nielsen, G. Rozenberg, and P. Thiagarajan. Elementary transition systems. In Theoretical Computer Science, volume 96, pages 3-33. Elsevier Science Publishers Ltd., 1992.

22. Ocean Observatories Initiative (OOI). http: //www. oceanobservatories.org/.

23. Savara JBoss Project. http://www. jboss .org/savara.

24. K. Takeuchi, K. Honda, and M. Kubo. An interaction-based language and its typing system. In PARLE'94, volume 817 of $L N C S$, pages 398-413. Springer, 1994.

25. J. Villard. Heaps and Hops. PhD thesis, ENS Cachan, 2011.

26. P. Wadler. Proposition as Sessions. In ICFP'12, pages 273-286, 2012.

\section{A Appendix for Section 3}

\section{A.1 Proof of Theorem 3.1}

Local Types Subtyping In order to relate global and local types, we define in Figure 2 a subtyping relation $\prec$ on local types. Local type $T^{\prime}$ is a super type of local type $T$, written $T \prec T^{\prime}$, if it offers more receive transitions. We note that $T_{i} \prec \sqcup_{i \in I} T_{i}$.

$$
\frac{\forall i \in I, T_{i} \prec T_{i}^{\prime}}{\mathrm{p} !\left\{a_{i} \cdot T_{i}\right\}_{i \in I} \prec \mathrm{p} !\left\{a_{i} \cdot T_{i}^{\prime}\right\}_{i \in I}} \quad \frac{I \subseteq J}{\mathrm{p} ?\left\{a_{i} \cdot T_{i}\right\}_{i \in I} \prec \mathrm{p} ?\left\{a_{j} \cdot T_{j}^{\prime}\right\}_{j \in J}} \quad \overline{\mathrm{t} \prec \mathrm{t}} \quad \frac{T \prec T^{\prime}}{\mu \mathrm{t} . T \prec \mu \mathrm{t} . T^{\prime}}
$$

Fig. 2. Subtyping between local types

This subtyping relation can be extended to configurations in the following way: $(\vec{T} ; \vec{w}) \prec\left(\vec{T}^{\prime} ; \vec{w}^{\prime}\right)$ if $\vec{w}=\vec{w}^{\prime}$ and $\forall \mathrm{p} \in \mathcal{P}, T_{\mathrm{p}} \prec T_{\mathrm{p}}^{\prime}$.

The main properties of subtyping is that it preserves traces, i.e. if $s \prec s^{\prime}$, then $s \approx s^{\prime}$. 
Extension of projection In order to prove Theorem 3.1, we extend the definition of projection to global intermediate states.

We represent the projected configuration $\llbracket G \rrbracket$ of a global type $G$ as a configuration $\left\{G\lceil\mathrm{p}\}_{\mathrm{p} \in \mathcal{P}}, \llbracket G \rrbracket_{\{\varepsilon\}_{\mathrm{qq}^{\prime} \in \mathcal{P}}}\right.$ where the content of the buffers $\llbracket G \rrbracket_{\{\varepsilon\}_{\mathrm{qq}^{\prime} \in \mathcal{P}}}$ is given by:

$$
\begin{aligned}
& \llbracket \mathrm{p} \rightsquigarrow \mathrm{p}^{\prime}: a_{j} \cdot G_{j} \rrbracket_{\left\{w_{\mathrm{qq}^{\prime}}\right\}_{\mathrm{qq}^{\prime} \in \mathcal{P}}}=\llbracket G_{j} \rrbracket_{\left\{w_{\mathrm{qq}^{\prime}}\right\}_{\mathrm{qq}^{\prime} \in \mathcal{P}}\left[w_{\mathrm{pp}^{\prime}}=w_{\mathrm{pp}^{\prime}} \cdot a_{j}\right]} \\
& \llbracket \mathrm{p} \rightarrow \mathrm{p}^{\prime}: a_{j} \cdot G_{j} \rrbracket_{\left\{w_{\mathrm{qq}^{\prime}}\right\}_{\mathrm{qq}^{\prime} \in \mathcal{P}}}=\llbracket G_{j} \rrbracket_{\left\{w_{\mathrm{qq}^{\prime}}\right\}_{\mathrm{qq}^{\prime} \in \mathcal{P}}} \\
& \llbracket \mathrm{p} \rightarrow \mathrm{p}^{\prime}:\left\{a_{j} \cdot G_{j}\right\}_{j \in J} \rrbracket_{\left\{w_{\mathrm{qq}^{\prime}}\right\}_{\mathrm{qq}^{\prime} \in \mathcal{P}}}=\llbracket G_{1} \rrbracket_{\left\{w_{\mathrm{qq}^{\prime}}\right\}_{\mathrm{qq}^{\prime} \in \mathcal{P}}} \\
& \llbracket \mu \text { t. } G \rrbracket_{\left\{w_{\mathrm{qq}^{\prime}}\right\}_{\mathrm{qq}^{\prime} \in \mathcal{P}}}=\left\{w_{\mathrm{qq}^{\prime}}\right\}_{\mathrm{qq}^{\prime} \in \mathcal{P}} \\
& \text { 【end } \rrbracket_{\left\{w_{\mathrm{qq}^{\prime}}\right\}_{\mathrm{qq}^{\prime} \in \mathcal{P}}}=\left\{w_{\mathrm{qq}^{\prime}}\right\}_{\mathrm{qq}^{\prime} \in \mathcal{P}}
\end{aligned}
$$

and where the projection algorithm $\uparrow \mathrm{q}$ is extended by:

$$
\mathrm{p} \rightsquigarrow \mathrm{p}^{\prime}: j\left\{a_{i} \cdot G_{i}\right\}_{i \in I}\left\lceil\mathrm{q}= \begin{cases}\mathrm{p} ?\left\{a_{i} \cdot G_{i}\lceil\mathrm{q}\}_{i \in I}\right. & \mathrm{q}=\mathrm{p}^{\prime} \\ G_{j}\lceil\mathrm{q} & \text { otherwise }\end{cases}\right.
$$

This extended projection allows us to match global type and projected local type transitions step by step.

Theorem 3.1 We prove Theorem 3.1 by combining the local type subtyping and extended projection into a step equivalence lemma. Theorem 3.1 is a simple consequence of Lemma A.1.

Lemma A.1 (Step equivalence). For all global type $G$ and local configuration $s$, if IG $\rrbracket \prec s$, then we have $G \stackrel{\ell}{\rightarrow} G^{\prime} \Leftrightarrow s \stackrel{\ell}{\rightarrow} s^{\prime}$ and $\llbracket G^{\prime} \rrbracket \prec s^{\prime}$.

Proof. The proof is by induction on the possible global and local transitions.

Correctness By induction on the structure of each reduction $G \stackrel{\ell}{\rightarrow} G^{\prime}$, we prove that $\llbracket G \rrbracket \stackrel{\ell}{\rightarrow} s$ with $\llbracket G^{\prime} \rrbracket \prec s$. We use the fact that if $s \prec s^{\prime}$, then $s \approx s^{\prime}$, to consider only matching transition for $\llbracket G \rrbracket$.

[GR1] where $G=\mathrm{p} \rightarrow \mathrm{p}^{\prime}:\left\{a_{i} \cdot G_{i}\right\}_{i \in I} \stackrel{\mathrm{pp}^{\prime} ! a_{i}}{\longrightarrow} G^{\prime}=\mathrm{p} \rightsquigarrow \mathrm{p}^{\prime}: j\left\{a_{i} \cdot G_{i}\right\}_{i \in I}$. The projection of $G$

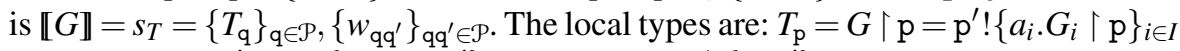
and $T_{\mathrm{p}^{\prime}}=G\left\lceil\mathrm{p}^{\prime}=\mathrm{p} ?\left\{a_{i} \cdot G_{i}\left\lceil\mathrm{p}^{\prime}\right\}_{i \in I}\right.\right.$ and (for $\mathrm{q} \notin\left\{\mathrm{p}, \mathrm{p}^{\prime}\right\}$ ) $T_{\mathrm{q}}=\sqcup_{i \in I} G_{i}\lceil\mathrm{q}$. Rule [LR 1] allows $\mathrm{p}^{\prime} !\left\{a_{i} . G_{i}\lceil\mathrm{p}\}_{i \in I} \stackrel{\mathrm{pp}^{\prime} ! a_{j}}{\longrightarrow} G_{j}\left\lceil\mathrm{p}\right.\right.$. We therefore have $s_{T} \stackrel{\mathrm{pp}^{\prime} ! a_{j}}{\longrightarrow}\left\{T_{\mathrm{q}}^{\prime}\right\}_{\mathrm{q} \in \mathcal{P}},\left\{w_{\mathrm{qq}^{\prime}}^{\prime}\right\}_{\mathrm{qq}^{\prime} \in \mathcal{P}}$, with $T_{\mathrm{q}}^{\prime}=T_{\mathrm{q}}$ if $\mathrm{q} \neq \mathrm{p}$, and $T_{\mathrm{p}}^{\prime}=G_{j} \uparrow \mathrm{p}$, and with $w_{\mathrm{qq}^{\prime}}^{\prime}=w_{\mathrm{qq}^{\prime}}$ if $\mathrm{qq}^{\prime} \neq \mathrm{pp}^{\prime}$, and $w_{\mathrm{pp}^{\prime}}^{\prime}=w_{\mathrm{pp}^{\prime}} \cdot a_{j}$.

Since $G_{j}\left\lceil\mathrm{q} \prec \sqcup_{i \in I} G_{i}\left\lceil\mathrm{q}\right.\right.$, we have $\left\{T_{\mathrm{q}}^{\prime}\right\}_{\mathrm{q} \in \mathcal{P}},\left\{w_{\mathrm{qq}^{\prime}}^{\prime}\right\}_{\mathrm{qq}^{\prime} \in \mathcal{P}} \prec \llbracket G \rrbracket$.

This corresponds exactly to the projection $\llbracket G^{\prime} \rrbracket$ of $G^{\prime}$.

[GR2] where $G=\mathrm{p} \rightsquigarrow \mathrm{p}^{\prime}: j\left\{a_{i} \cdot G_{i}\right\}_{i \in I} \stackrel{\mathrm{pp}^{\prime} ? a_{j}}{\longrightarrow} G^{\prime}=G_{j}$. The projection of $G$ is $\llbracket G \rrbracket=s_{T}=$

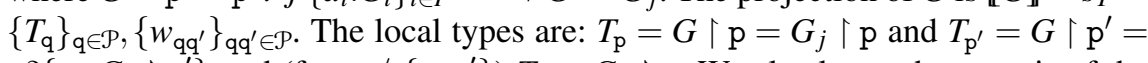
$\mathrm{p} ?\left\{a_{j} \cdot G_{j}\left\lceil\mathrm{p}^{\prime}\right\}\right.$ and (for $\left.\mathrm{q} \notin\left\{\mathrm{p}, \mathrm{p}^{\prime}\right\}\right) T_{\mathrm{q}}=G_{j}\left\lceil\mathrm{q}\right.$. We also know that $w_{\mathrm{pp}^{\prime}}$ is of the form $w_{\mathrm{pp}}^{\prime} \cdot a_{j}$.

Using [LR2], $\left\{T_{\mathrm{q}}\right\}_{\mathrm{q} \in \mathcal{P}},\left\{w_{\mathrm{qq}^{\prime}}\right\}_{\mathrm{qq}^{\prime} \in \mathcal{P}} \stackrel{\mathrm{pp}^{\prime} ? a_{j}}{\longrightarrow}\left\{G_{j}\lceil\mathrm{q}\}_{\mathrm{q} \in \mathcal{P}},\left\{w_{\mathrm{qq}^{\prime}}^{\prime}\right\}_{\mathrm{qq}^{\prime} \in \mathcal{P}}\right.$ with $w_{\mathrm{qq}^{\prime}}^{\prime}=w_{\mathrm{qq}^{\prime}}$ if $\mathrm{qq}^{\prime} \neq \mathrm{pp}^{\prime}$. The result of the transition is the same as the projection $\llbracket G^{\prime} \rrbracket$ of $G^{\prime}$. 
[GR3] where $G=\mu$ t. $G^{\prime} \stackrel{\ell}{\rightarrow} G^{\prime \prime}$.

By hypothesis, we know that $G^{\prime}\left[\mathrm{t} / \mu \mathrm{t} . G^{\prime}\right] \stackrel{\ell}{\rightarrow} G^{\prime \prime}$. By induction, we know that $\llbracket G^{\prime}\left[\mathrm{t} / \mu \mathrm{t} . G^{\prime}\right] \rrbracket=$ $s_{T}=\left\{T_{\mathrm{q}}\right\}_{\mathrm{q} \in \mathcal{P}},\left\{w_{\mathrm{qq}^{\prime}}\right\}_{\mathrm{qq}^{\prime} \in \mathcal{P}}$ can do a reduction $\stackrel{\ell}{\rightarrow}$ to $\llbracket G^{\prime \prime} \rrbracket=s_{T}=\left\{T_{\mathrm{q}}^{\prime}\right\}_{\mathrm{q} \in \mathcal{P}},\left\{w_{\mathrm{qq}^{\prime}}^{\prime}\right\}_{\mathrm{qq}^{\prime} \in \mathcal{P} \text {. }}$ Projection is homomorphic for recursion, hence $G^{\prime}\left[\mu \mathrm{t} . G^{\prime} / \mathrm{t}\right]\left\lceil\mathrm{q}=G^{\prime}\left\lceil\mathrm{q}\left[\mu \mathrm{t} . G^{\prime} \mid \mathrm{q} / \mathrm{t}\right]\right.\right.$. We use [LR4] to conclude.

[GR4] where $\mathrm{p} \rightarrow \mathrm{q}:\left\{a_{i} \cdot G_{i}\right\}_{i \in I} \stackrel{\ell}{\rightarrow} \mathrm{p} \rightarrow \mathrm{q}:\left\{a_{i} \cdot G_{i}^{\prime}\right\}_{i \in I}$ and $\mathrm{p}, \mathrm{q} \notin \operatorname{subj}(\ell)$. By induction, we know that, $\forall i \in I, \llbracket G_{i} \rrbracket \stackrel{\ell}{\rightarrow} \llbracket G_{i}^{\prime} \rrbracket$. We need to prove that $\llbracket \mathrm{p} \rightarrow \mathrm{q}:\left\{a_{i} \cdot G_{i}\right\}_{i \in I} \rrbracket \stackrel{\ell}{\rightarrow}$ $\llbracket \mathrm{p} \rightarrow \mathrm{q}:\left\{a_{i} \cdot G_{i}^{\prime}\right\}_{i \in I} \rrbracket$. The projections for all participants are identical, except for $\mathrm{q}^{\prime}=\operatorname{subj}(\ell)$, whose projection is (computed by merging) $\sqcup_{i \in I} G_{i}\left\lceil\mathrm{q}^{\prime}\right.$. Since $\forall i \in$ $I, \llbracket G_{i} \rrbracket \stackrel{\ell}{\rightarrow} \llbracket G_{i}^{\prime} \rrbracket$, we know that all the $G_{i}\left\lceil\mathrm{q}^{\prime}\right.$ have at least the prefix corresponding to $\ell$, and that, using either [LR1] or [LR2], the continuations are the $G_{i}^{\prime}\left\lceil\mathrm{q}^{\prime}\right.$. We can then conclude that the $\sqcup_{i \in I} G_{i}\left\lceil\mathrm{q}^{\prime} \stackrel{\ell}{\rightarrow} \sqcup_{i \in I} G_{i}^{\prime}\left\lceil\mathrm{q}^{\prime}\right.\right.$.

[GR5] where $\mathrm{p} \rightsquigarrow \mathrm{q}: j\left\{a_{i} \cdot G_{i}\right\}_{i \in I} \stackrel{\ell}{\rightarrow} \mathrm{p} \rightsquigarrow \mathrm{q}: j\left\{a_{i} \cdot G_{i}^{\prime}\right\}_{i \in I}$ and $\mathrm{q} \notin \operatorname{subj}(\ell)$ with $G_{i}^{\prime}=G_{i}$ for $i \neq j$. By induction, we know that, $\llbracket G_{j} \rrbracket \stackrel{\ell}{\rightarrow} \llbracket G_{j}^{\prime} \rrbracket$. We need to prove that $\llbracket \mathrm{p} \rightsquigarrow$ $\mathrm{q}: j\left\{a_{i} \cdot G_{i}\right\}_{i \in I} \rrbracket \stackrel{\ell}{\rightarrow} \llbracket \mathrm{p} \rightarrow \mathrm{q}:\left\{j . U_{i}\right\}_{G_{i}^{i}} i \in I \rrbracket$. The projections for all participants are identical, except for $\mathrm{q}^{\prime}=\operatorname{subj}(\ell)$, whose projection is $G_{j}\left\lceil\mathrm{q}^{\prime}\right.$. By induction, $\left.G_{j}\right\rceil$ $\mathrm{q}^{\prime} \stackrel{\ell}{\rightarrow} G_{j}^{\prime}\left\lceil\mathrm{q}^{\prime}\right.$, which allows us to conclude.

Completeness We prove by induction on $\llbracket G \rrbracket=$

$$
\left\{T_{\mathrm{p}}\right\}_{\mathrm{p} \in \mathcal{P}},\left\{w_{\mathrm{qq}^{\prime}}\right\}_{\mathrm{qq}^{\prime} \in \mathcal{P}} \stackrel{\ell}{\rightarrow}\left\{T_{\mathrm{p}}^{\prime}\right\}_{\mathrm{p} \in \mathcal{P}},\left\{w_{\mathrm{qq}^{\prime}}^{\prime}\right\}_{\mathrm{qq}^{\prime} \in \mathcal{P}} \text { that } G \stackrel{\ell}{\rightarrow} G^{\prime} \text { with } \llbracket G^{\prime} \rrbracket \prec\left\{T_{\mathrm{p}}^{\prime}\right\}_{\mathrm{p} \in \mathcal{P}},\left\{w_{\mathrm{qq}^{\prime}}^{\prime}\right\}_{\mathrm{qq}^{\prime} \in \mathcal{P} \text {. }}
$$

[LR1] There is $T_{\mathrm{p}}=G\left\lceil\mathrm{p}=\mathrm{p}^{\prime} !\left\{a_{i} \cdot G_{i}\lceil\mathrm{p}\}_{i \in I}\right.\right.$. By definition of projection, $G$ has $\mathrm{p} \rightarrow$ q: $\left\{a_{i} \cdot G_{i}\right\}_{i \in I}$ as subterm, possibly several times (by mergeability). By definition of projection, we note that no action in $G$ can involve p before any of the occurrences of $\mathrm{p} \rightarrow \mathrm{q}:\left\{a_{i} \cdot G_{i}\right\}_{i \in I}$. Therefore we can apply as many times as needed [GR4] and [GR5], and use [GR1] to reduce to $\mathrm{p} \rightsquigarrow \mathrm{q}: a_{j} . G_{j}$. The projection of the resulting global type corresponds to a subtype to the result of [LR1].

[LR2] There is $T_{\mathrm{p}}=G \mid \mathrm{p}=\mathrm{q} ?\left\{a_{j} . G_{j} \mid \mathrm{p}\right\}_{j \in J}$. To activate [LR2], there should be a value $a_{j}$ in the buffer $w_{\mathrm{pq}}$. By definition of projection, $G$ has therefore $\mathrm{p} \rightsquigarrow \mathrm{q}: j\left\{a_{i} \cdot G_{i}\right\}_{i \in I}$ as subterm, possibly several times (by mergeability). By definition of projection, no action in $G$ can involve $\mathrm{p}$ before any of the occurrences of $\mathrm{p} \rightsquigarrow \mathrm{q}: j\left\{a_{i} \cdot G_{i}\right\}_{i \in I}$. We can apply as many times as needed [GR4] and [GR5] and use [GR2] to reduce to $G_{j}$. The projection of the resulting global type corresponds to the result of [LR2].

[LR3] where $T=\mu \mathrm{t} . T^{\prime}$. Projection is homomorphic with respect to recursion. Therefore $G$ is of the same form. We can use [GR3] and induction to conclude.

\section{A.2 Local types and CFSMs}

Proposition 3.1 For the determinism, we note that all $a_{i}$ in $\mathrm{p} ?\left\{a_{i} \cdot T_{i}\right\}_{i \in I}$ and $\mathrm{p} !\left\{a_{i} \cdot T_{i}\right\}_{i \in I}$ are distinct. Directdness is by the syntax of branching and selection types. Finally, for non-mixed states, we can check a state is either sending or receiving state as one state represents either branching and selection type.

Proposition 3.2 The first clause is by the induction of $M$ using the translation of $\mathcal{T}$. The second clause is by the induction of $T$ using the translation of $\mathcal{A}$. Both are mechanical. 


\section{B Appendix for Section 4}

We say that a configuration $s$ with $t_{1}$ and $t_{2}$ satisfies the one-step diamond property if, assuming $s \stackrel{t_{1}}{\rightarrow} s_{1}$ and $s \stackrel{t_{2}}{\rightarrow} s_{2}$ with $t_{1} \neq t_{2}$, there exists $s^{\prime}$ such that $s_{1} \stackrel{t_{\rightarrow}^{\prime}}{\rightarrow} s^{\prime}$ and $s_{2} \stackrel{t_{2}^{\prime}}{\rightarrow} s^{\prime}$ where $\operatorname{act}\left(t_{1}\right)=\operatorname{act}\left(t_{2}^{\prime}\right)$ and $\operatorname{act}\left(t_{2}\right)=\operatorname{act}\left(t_{1}^{\prime}\right)$. We use the following lemma to permute the two actions.

Lemma B.1 (diamond property in basic machines). Suppose $S=\left(M_{\mathrm{p}}\right)_{\mathrm{p} \in \mathcal{P}}$ and $S$ is basic. Assume $s \in R S(S)$ and $s \stackrel{t_{1}}{\rightarrow} s_{1}$ and $s \stackrel{t_{2}}{\rightarrow} s_{2}$.

1. If $t_{1}$ and $t_{2}$ are both sending actions such that act $\left(t_{1}\right)=\mathrm{p}_{1} \mathrm{q}_{1} ! a_{1}$ and $\operatorname{act}\left(t_{2}\right)=$ $\mathrm{p}_{2} \mathrm{q}_{2} ! a_{2}$, we have either:

(a) $\mathrm{p}_{1}=\mathrm{p}_{2}$ and $\mathrm{q}_{1}=\mathrm{q}_{2}$ and $a_{1}=a_{2}$ with $s_{1}=s_{2}$;

(b) $\mathrm{p}_{1}=\mathrm{p}_{2}$ and $\mathrm{q}_{1}=\mathrm{q}_{2}$ and $a_{1} \neq a_{2}$;

(c) $\mathrm{p}_{1} \neq \mathrm{p}_{2}$ and $\mathrm{q}_{1} \neq \mathrm{q}_{2}$ with $a_{1} \neq a_{2}$, and $s$ with $t_{1}$ and $t_{2}$ satisfies the diamond property.

2. If $t_{1}$ and $t_{2}$ are both receiving actions such that act $\left(t_{1}\right)=\mathrm{p}_{1} \mathrm{q}_{1}$ ? $a_{1}$ and act $\left(t_{2}\right)=$ $\mathrm{p}_{2} \mathrm{q}_{2} ? a_{2}$, we have either:

(a) $\mathrm{p}_{1}=\mathrm{p}_{2}$ and $\mathrm{q}_{1}=\mathrm{q}_{2}$ and $a_{1}=a_{2}$ with $s_{1}=s_{2}$;

(b) $\mathrm{p}_{1} \neq \mathrm{p}_{2}$ and $\mathrm{q}_{1} \neq \mathrm{q}_{2}$ with $s_{1} \neq s_{2}$, and $s$ with $t_{1}$ and $t_{2}$ satisfies the diamond property.

3. If $t_{1}$ is a receiving action and $t_{2}$ is a sending action such that act $\left(t_{1}\right)=\mathrm{p}_{1} \mathrm{q}_{1} ? a_{1}$ and $\operatorname{act}\left(t_{2}\right)=\mathrm{p}_{2} \mathrm{q}_{2} ! a_{2}$, we have either:

(a) $\mathrm{q}_{1}=\mathrm{q}_{2}$ and $\mathrm{p}_{1} \neq \mathrm{p}_{2}$; or

(b) $\mathrm{p}_{1}=\mathrm{p}_{2}$ and $\mathrm{q}_{1} \neq \mathrm{q}_{2}$; or

(c) $\mathrm{p}_{1} \neq \mathrm{p}_{2}$ and $\mathrm{q}_{1} \neq \mathrm{q}_{2}$

with $s_{1} \neq s_{2}$, and $s$ with $t_{1}$ and $t_{2}$ satisfies the diamond property.

Proof. For (1), there is no case such that $\mathrm{p}_{1} \neq \mathrm{p}_{2}$ and $\mathrm{q}_{1}=\mathrm{q}_{2}$ since $S$ is directed. Then if $\mathrm{p}_{1}=\mathrm{p}_{2}$ and $\mathrm{q}_{1}=\mathrm{q}_{2}$ and $a_{1}=a_{2}$, then $s_{1}=s_{2}$ by the determinism. For (2), there is no case such that $\mathrm{p}_{1} \neq \mathrm{p}_{2}$ and $\mathrm{q}_{1}=\mathrm{q}_{2}$ since $S$ is directed. Also there is no case such that $\mathrm{p}_{1}=\mathrm{p}_{2}$ and $\mathrm{q}_{1}=\mathrm{q}_{2}$ and $a_{1} \neq a_{2}$ since the communication between the same peer is done via an FIFO queue. For (3), there is no case such that $q_{1}=q_{2}$ and $p_{1}=p_{2}$ because of no-mixed state.

The following definition aims to explicitly describe the causality relation between the actions. These are useful to identify the permutable actions.

\section{Definition B.1 (causality).}

1. Suppose $s_{0} \stackrel{\varphi}{\rightarrow} s$ and $\varphi=\varphi_{0} \cdot t_{1} \cdot \varphi_{1} \cdot t_{2} \cdot \varphi_{2}$. We write $t_{1} \triangleleft t_{2}\left(t_{2}\right.$ depends on $\left.t_{1}\right)$ if either (1) $t_{1}=\mathrm{pq} ! a$ and $t_{1}=\mathrm{pq} ? a$ for some p and q or (2) $\operatorname{subj}\left(t_{1}\right)=\operatorname{subj}\left(t_{2}\right)$.

2. We say $\varphi=t_{0} \cdot t_{1} \cdot t_{2} \cdots t_{n}$ is the causal chain if $s_{0} \stackrel{\varphi^{\prime}}{\rightarrow} s^{\prime}$ and $\varphi \subseteq \varphi^{\prime}$ with, for all $0 \leq k \leq n-1$, there exists $i$ such that $i>k$ and $t_{k} \triangleleft t_{i}$. We call $\varphi$ the maximum causal chain if there is no causal chain $\varphi^{\prime \prime}$ such that $\varphi \subsetneq \varphi^{\prime \prime} \subseteq \varphi^{\prime}$.

3. Suppose $s_{0}{ }_{\rightarrow} s$ and $\varphi=\varphi_{0} \cdot t_{1} \cdot \varphi_{1} \cdot t_{2} \cdot \varphi_{2}$. We write $t_{i} \sharp t_{j}$ if there is no causal chain from $t_{i}$ to $t_{j}$ with $i<j$.

By Lemma B.1, we have:

Lemma B.2 (maximum causality). Suppose $S$ is basic and $s \in R S(S)$. Then for all $s \stackrel{\varphi}{\rightarrow} s^{\prime}$, we have $s \stackrel{\varphi_{m} \cdot \varphi^{\prime \prime}}{\longrightarrow} s^{\prime}$ and $s \stackrel{\varphi^{\prime \prime} \cdot \varphi_{m}^{\prime}}{s^{\prime}}$ where $\varphi_{m}, \varphi_{m}^{\prime}$ are the maximum causal chain. 
Lemma B.3 (output-input dependency). Suppose $S$ is basic. Then there is no causal chain $t_{0} \cdot t_{1} \cdot t_{2} \cdots t_{n}$ such that act $\left(t_{0}\right)=\mathrm{pq} ! a$ and $\operatorname{act}\left(t_{n}\right)=\mathrm{pq}^{\prime} ? b$ with $a \neq b$ and $\operatorname{act}\left(t_{i}\right) \neq$ $\mathrm{pq}$ ?c for any $c(1 \leq i \leq n-1)$.

Proof. We use the following definition. The causal chain $\varphi=t_{0} \cdot t_{1} \cdots t_{n}$ is called

1. O-causal chain if for all $1 \leq i \leq n, t_{i}=\mathrm{pq}_{i} ! a_{i}$ with some $\mathrm{q}_{i}$ and $a_{i}$.

2. I-causal chain if for all $1 \leq i \leq n, t_{i}=\mathrm{q}_{i} \mathrm{p}$ ? $a_{i}$ with some $\mathrm{q}_{i}$ and $a_{i}$.

Then any single causal chain $\varphi=\tilde{t}_{0} \cdot \tilde{t}_{1} \cdots \tilde{t}_{n}$ can be decomposed into alternating $\mathrm{O}$ and I causal chains where $t_{i}=\cdot t_{i 0} \cdots t_{i n_{i}}$ with either (1) $a c t\left(t_{i n_{i}}\right)=\mathrm{pq} ! a$ and $a c t\left(t_{i+10}\right)=\mathrm{q}^{\prime} \mathrm{p} ? b$; (2) $\operatorname{act}\left(t_{i n_{i}}\right)=\mathrm{pq} ? a$ and $a c t\left(t_{i+10}\right)=\mathrm{qp}^{\prime} ! b$; or (3) $\operatorname{act}\left(t_{i n_{i}}\right)=\mathrm{pq} ! a$ and $\operatorname{act}\left(t_{i+10}\right)=\mathrm{pq} ? a$. In the case of $(1,2)$, we note $\operatorname{subj}\left(t_{i h}\right)=\operatorname{subj}\left(t_{i+1 k}\right)$ for all $0 \leq h \leq n_{i}$ and $0 \leq k \leq n_{i+1}$.

Now assume $S$ is basic and there is a sequence $\varphi=t_{0} \cdot t_{1} \cdots t_{n}$ such that $\operatorname{act}\left(t_{0}\right)=$ $\mathrm{p}_{0} \mathrm{q}_{0} ! a_{0}$ and $a c t\left(t_{n}\right)=\mathrm{p}_{n} \mathrm{q}_{n}$ ? $a_{n}$ with $\mathrm{p}_{0}=\mathrm{q}_{n}, a_{0} \neq a_{n}$ and $a c t\left(t_{i}\right) \neq \mathrm{p}_{0} \mathrm{q}_{0}$ ? $a$ for any $a$ $(1 \leq i \leq n-1)$. We prove $\varphi$ is not a causal chain by the induction of the length of $\varphi$.

Case $n=1$. By definition, $t_{0} \sharp t_{n}$.

Case $n>1$. If $\varphi$ is a causal chain, there is a decomposition into $\mathrm{O}$ and I causal chains such that $\varphi=\tilde{t}_{0} \cdot \tilde{t}_{1} \cdots \tilde{t}_{m}$ where $t_{i}=t_{i 0} \cdots t_{i n_{i}}$. By the condition $t_{i} \neq \mathrm{p}_{0} \mathrm{q}_{0}$ ? $a$ for any $a$ $(1 \leq i \leq n-1)$, the case (3) above is excluded. Hence we have $\operatorname{subj}\left(t_{i h}\right)=\operatorname{subj}\left(t_{i+1 k}\right)$ for all $0 \leq h \leq n_{i}$ and $0 \leq k \leq n_{i+1}$. This implies

1. $\mathrm{p}_{0}=\mathrm{p}_{i j}$ with $i$ even (in the $\mathrm{O}$ causal chains)

2. $\mathrm{q}_{i j}=\mathrm{q}_{0}$ with $i$ odd (in the I causal chains); and

3. $\mathrm{p}_{i n_{i}}=\mathrm{q}_{i+10}$ with $i$ even.

This implies $\mathrm{p}_{0}=\mathrm{q}_{0}$ which contradicts the definition of the channels of CFSMs (i.e. $\mathrm{p}_{0} \neq \mathrm{q}_{0}$ if $\mathrm{p}_{0} \mathrm{q}_{0}$ is a channel). Hence there is no causal chain from $\operatorname{act}\left(t_{0}\right)=\mathrm{p}_{0} \mathrm{q}_{0} ! a_{0}$ to $\operatorname{act}\left(t_{n}\right)=\mathrm{p}_{0} \mathrm{q}_{0} ? a_{n}$ if $\operatorname{act}\left(t_{i}\right) \neq \mathrm{p}_{0} \mathrm{q}_{0} ? a$ and $a_{0} \neq a_{n}$.

Lemma B.4 (input availablity). Assume $S=\left(M_{\mathrm{p}}\right)_{\mathrm{p} \in \mathcal{P}}$ is basic and multiparty compatible. Then for all $s \in R S(S)$, if $s \stackrel{\mathrm{pp}^{\prime} ! a_{1}}{\rightarrow} s^{\prime}$, then $s^{\prime} \varphi_{\rightarrow} s_{2} \stackrel{\mathrm{pp}}{\longrightarrow} ? a_{3}$.

Proof. We use Lemma B.1 and Lemma B.2. Suppose $s \in R S(S)$ and $s \stackrel{t}{\rightarrow} s^{\prime}$ such that $\operatorname{act}(t)=\mathrm{pp}^{\prime} ! a$. By contradiction, assume there is no $\varphi^{\prime}$ such that $s^{\prime} \stackrel{\varphi^{\prime}}{\rightarrow} \stackrel{t^{\prime}}{\rightarrow} s^{\prime \prime}$ with act $(t)=$ $\mathrm{pp}^{\prime} ? a$. Then there should be some input state $\left(q, \mathrm{qp}^{\prime} ? b, q^{\prime}\right) \in \delta_{\mathrm{p}^{\prime}}$ where $q \stackrel{\mathrm{qp}^{\prime} ? b^{\prime}}{\longrightarrow} q^{\prime \prime} \stackrel{p_{1}}{\longrightarrow} \stackrel{\mathrm{pp}^{\prime} ? a}{\longrightarrow} q^{\prime \prime \prime}$ where $b \neq b^{\prime}$ (hence $q^{\prime} \neq q^{\prime \prime}$ by determinism), i.e. $\mathrm{qp}^{\prime} ? b$ leads to an incompatible path with one lead to the action $\mathrm{qp}^{\prime} ? a$.

Suppose $s^{\prime} \varphi_{0} \rightarrow \frac{t_{b i}}{\rightarrow} s^{\prime \prime}$ with $t_{b i}=\left(q, \mathrm{qp}^{\prime} ? b, q^{\prime}\right)$. Then $\varphi_{0}$ should include the corresponding output action $a c t\left(t_{b o}\right)=\mathrm{qp}^{\prime} ! b$. By Lemma B.2, without loss of generality, we assume $\varphi_{0} \cdot t_{b i}$ is the maximum causal chain to $t_{b i}$. Let us write $\varphi_{0}=t_{0} \triangleleft t_{1} \triangleleft \cdots \triangleleft t_{n}$. By Lemma B.1, we can set $t_{b o}=t_{n}$. Note that for all $i$, act $\left(t_{i}\right) \neq \mathrm{pp}^{\prime} ? a^{\prime}$ by the assumption: since if $a c t\left(t_{i}\right) \neq \mathrm{pp}^{\prime} ? a$, then it contradicts the assumption such that $t$ does not have a corresponding input; and if $a c t\left(t_{i}\right)=\mathrm{pp}^{\prime} ? a^{\prime}$ with $a \neq a^{\prime}$ then, by directedness of $S$, it contradicts to the assumption that $t_{b i}$ is the first input which leads to the incompatible path. Then there are three cases.

1. there is a chain from $t$ to $t_{n}=t_{b o}$, i.e. there exists $0 \leq i \leq n$ such that $t \triangleleft t_{i} \triangleleft \cdots \triangleleft t_{n}$.

2. there is no direct chain from $t$ to $t_{n}$ but there is a chain to $t_{b i}$, i.e. there exists $0 \leq i \leq n$ such that $t \triangleleft t_{i} \triangleleft \cdots \triangleleft t_{b i}$. 
3. there is no chain from $t$ to either $t_{n}$ or $t_{b i}$.

Case 1: By the assumption, there is no $t_{j}$ such that $a c t\left(t_{j}\right)=\mathrm{pp}^{\prime} ? a^{\prime}$. Hence $t_{i}=\mathrm{pp}^{\prime \prime} ! a^{\prime}$ for some $a^{\prime}$ and $\mathrm{p}^{\prime \prime}$.

Case 1-1: there is no input in $t_{j}$ in $t \triangleleft t_{i} \triangleleft \cdots \triangleleft t_{n-1}$. Then $\mathrm{p}=\mathrm{q}$, i.e. $\mathrm{qp}^{\prime} ! b=\mathrm{pp}^{\prime} ! b$. Then by the definition of $s \stackrel{t}{\rightarrow} s^{\prime}$ (i.e. by FIFO semantics at each channel), $\mathrm{pp}^{\prime} ? b$ cannot perform before $\mathrm{pp}^{\prime} ? a$. This case contradicts to the assumption $\mathrm{pp}^{\prime} ? a$ is not available.

Case 1-2: there is an input $t_{j}$ in $t \triangleleft t_{i} \triangleleft \cdots \triangleleft t_{n-1}$. By $t \triangleleft t_{i}, \operatorname{subj}\left(\operatorname{act}\left(t_{i}\right)\right)=\mathrm{p}$. Hence we have either $\operatorname{act}\left(t_{i}\right)=\mathrm{pq}_{i} ! a_{i}$ with $\mathrm{q} \neq \mathrm{q}_{i}$ or $\operatorname{act}\left(t_{i}\right)=\mathrm{q}_{i} \mathrm{p} ? a_{i}$.

Case 1-2-1: $\operatorname{act}\left(t_{i}\right)=\mathrm{pq}_{i} ! a_{i}$. Then there is a path $q \stackrel{\mathrm{pq} ! a}{\longrightarrow} \stackrel{\mathrm{pq}_{i} ! a_{i}}{\longrightarrow} q^{\prime}$ in $M_{\mathrm{p}}$. Hence by the multiparty compatibility, there should be the traces $\mathrm{pq} ? a \cdot \varphi \cdot \mathrm{pq}_{i} ? a_{i}$ with $\varphi$ alternation from the machine with respect to $\left\{M_{\mathrm{r}}\right\}_{\mathrm{r} \in \mathcal{P} \backslash \mathrm{p}}$. This contradicts to the assumption that $\mathrm{pp}^{\prime} ? a$ is not available.

Case 1-2-2: $\operatorname{act}\left(t_{i}\right)=\mathrm{q}_{i} \mathrm{p}$ ? $a_{i}$. Similarly with the case Case 1-2-1, by the multiparty compatibility, there should be the traces $\mathrm{pq} ? a \cdot \varphi \cdot \mathrm{pq}_{i} ? a_{i}$ with $\varphi$ alternation from the machine with respect to $\left\{M_{\mathrm{r}}\right\}_{\mathrm{r} \in \mathcal{P} \backslash \mathrm{p}}$. Hence it contradicts to the assumption.

Case 2: Assume the chain such that $t \triangleleft t_{i} \triangleleft \cdots \triangleleft t_{b i}$ and $t \sharp t_{n}$. As the same reasoning as Case $1, \mathrm{p} \neq \mathrm{q}$ and $t_{i}$ is either $\mathrm{pq}_{i} ! a_{i}$ or $\mathrm{q}_{i} \mathrm{p} ? a_{i}$. Then we use the multiparty compatibility.

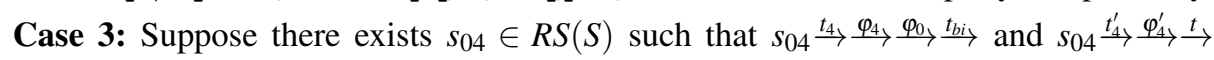
where $t_{4}$ leads to $t_{b i}$ and $t_{4}^{\prime}$ leads to $t$.

Case 3-1: Suppose $t_{4}$ and $t_{4}^{\prime}$ are both sending actions. By Lemma B.1, there are three cases.

(a) This case does not satisfy the assumption since $s_{1}=s_{2}$.

(b) We set $\operatorname{act}\left(t_{4}\right)=\mathrm{p}_{4} \mathrm{q}_{4} ! d$ and $\operatorname{act}\left(t_{4}^{\prime}\right)=\mathrm{p}_{4} \mathrm{q}_{4} ! d^{\prime}$ with $d \neq d^{\prime}$. In this case, we cannot execute both $t$ and $t_{b i}$. Hence there is no possible way to execute $t_{b i}$. This contradicts to the assumption.

(c) Since this case satisfy the diamond property, we apply the same routine from $s^{\prime}$ such that $s_{04} \stackrel{t_{4}}{\longrightarrow} \stackrel{t_{4} 1}{\longrightarrow} s^{\prime}$ and $s_{04} \stackrel{t_{4}^{\prime}}{\longrightarrow} \stackrel{t_{4} 2}{\longrightarrow} s^{\prime}$ and $\operatorname{act}\left(t_{4}\right)=\stackrel{t_{4} 2}{\longrightarrow}$ and $\operatorname{act}\left(t_{4}^{\prime}\right)=\stackrel{t_{4} 1}{\longrightarrow}$ where the length of the sequences to $t$ and $t_{b i}$ is reduced (hence this case is eventually matched with other cases).

Case 3-2: Suppose $t_{4}$ and $t_{4}^{\prime}$ are both sending actions. By Lemma B.1, there are two cases. The case (a) is as the same as the case 3-1-(b) and the case (b) is as the same as the case 3-1-(c).

Case 3-3: Suppose $t_{4}$ is a sending action and $t_{4}^{\prime}$ is receiving action. This case is as the same as the case 3-1-(c) and This concludes the proof.

We can extend the above lemma. The proof is similar.

Lemma B.5 (general input availablity). Assume $S=\left(M_{\mathrm{p}}\right)_{\mathrm{p} \in \mathcal{P}}$ is basic and multiparty compatible. Then for all $s \in R S(S)$, if $s \stackrel{\mathrm{pp}^{\prime} ! a_{3}}{\rightarrow} s_{1} \varphi_{\rightarrow} s^{\prime}$ with $\mathrm{pp}^{\prime} ? a \notin \varphi$, then $s^{\prime} \stackrel{\varphi^{\prime}}{\rightarrow} s_{2} \stackrel{\mathrm{pp}^{\prime} ? a}{\longrightarrow} s_{3}$.

\section{B.1 Proofs of Theorem 4.1}

We first prove the following stable property.

Proposition B.1 (stable property). Assume $S=\left(M_{\mathrm{p}}\right)_{\mathrm{p} \in \mathcal{P}}$ is basic and multiparty compatible. Then $S$ satisfies the stable property, i.e. if, for all $s \in R S(S)$, there exists an execution $\stackrel{\varphi^{\prime}}{\rightarrow}$ such that $s \stackrel{\varphi^{\prime}}{\rightarrow} s^{\prime}$ and $s^{\prime}$ is stable, and there is a 1-bounded execution $s_{0} \stackrel{\varphi^{\prime \prime}}{\rightarrow} s^{\prime}$. 
Proof. We proceed by the induction of the total number of messages (sending actions) which should be closed by the corresponding received actions. Once all messages are closed, we can obtain 1-bound execution.

Suppose $s_{1}, s_{2}$ are the states such that $s_{0} \stackrel{\varphi_{1}}{\longrightarrow} s_{1} \stackrel{t_{1}}{\rightarrow} s_{2} \underline{\varphi}_{\longrightarrow}^{\prime} s^{\prime}$ where $\varphi_{1}$ is a 1-bounded execution and $s_{1} \stackrel{t_{1}}{\rightarrow} s_{2}$ is the first transition which is not followed by the corresponding received action. Since $\varphi_{1}$ is a 1-bounded execution, there is $s_{3}$ such that $s_{2}{ }_{2} \rightarrow s_{3}$ where $t_{1}$ and $t_{2}$ are both sending actions. Then by the definition of the compatibility and Lemma B.4, we have

$$
s_{1} \stackrel{t_{1}}{\rightarrow} s_{2} \stackrel{\varphi_{2}}{\longrightarrow} \stackrel{\overline{t_{1}}}{\longrightarrow} s_{3}^{\prime}
$$

where $\varphi_{2}$ is an alternation execution and $\overline{t_{1}}=\mathrm{pq} ? a$. Assume $\varphi_{2}$ is a minimum execution which leads to $\overline{t_{1}}$. We need to show

$$
s_{1} \stackrel{\varphi_{2}}{\rightarrow} \stackrel{t_{1}}{\longrightarrow} \stackrel{\overline{t_{1}}}{\longrightarrow} s_{3}^{\prime} \stackrel{t_{2}}{\rightarrow} s_{4}
$$

Then we can apply the same routine for $t_{2}$ to close it by the corresponding receiving action $\overline{t_{2}}$. Applying this to the next sending state one by one, we can reach an 1-bounded execution. Let $\varphi_{2}=t_{4} \cdot \varphi_{2}^{\prime}$. Then by the definition of multiparty compatibility, $\operatorname{act}\left(t_{4}\right)=$ $\mathrm{p}^{\prime} \mathrm{q}^{\prime} ! c$ and $\mathrm{p}^{\prime} \neq \mathrm{p}$ and $\mathrm{q}^{\prime} \neq \mathrm{q}$. Hence by Lemma B.1(1), there exists the execution such that

$$
s_{1} \stackrel{t_{4}}{\rightarrow} \stackrel{t_{1}}{\rightarrow} \stackrel{\varphi_{2}^{\prime}}{\longrightarrow} \stackrel{\overline{t_{1}}}{\longrightarrow} s_{3}^{\prime} \stackrel{t_{2}}{\rightarrow} s_{4}
$$

Let $\varphi_{2}^{\prime}=\overline{t_{4}} \cdot \varphi_{2}^{\prime \prime}$ where $\overline{t_{1}}=\mathrm{p}^{\prime} \mathrm{q}^{\prime} ? c$. Then this time, by Lemma B.1(2), we have:

$$
s_{1} \stackrel{t_{4}}{\rightarrow} \stackrel{\overline{t_{4}}}{\rightarrow} \stackrel{t_{1}}{\rightarrow} \stackrel{\varphi_{2}^{\prime \prime}}{\longrightarrow} \stackrel{\overline{t_{1}}}{\longrightarrow} s_{3}^{\prime} \stackrel{t_{2}}{\rightarrow} s_{4}
$$

where $\varphi_{1} \cdot t_{4} \cdot \overline{t_{4}}$ is a 1 -bounded execution. Applying this permutation repeatedly, we have

$$
s_{1} \stackrel{\varphi_{3}}{\longrightarrow} \stackrel{t_{1}}{\rightarrow} \stackrel{\overline{t_{1}}}{\rightarrow} s_{3}^{\prime} \stackrel{t_{2}}{\rightarrow} s_{4}
$$

where $\varphi_{3}$ is an 1-bounded execution. We apply the same routine for $t_{2}$ and conclude $s_{1} \stackrel{\varphi^{\prime}}{\rightarrow} s^{\prime}$ for some stable $s^{\prime}$.

From the stable property, the orphan message- and the reception error-freedom are immediate. Also the liveness is a corollary by the orphan message- and deadlockfreedom. Hence we only prove the deadlock-freedom assuming the stable property.

Deadlock-freedom Assume $S$ is basic and satisfy the multiparty session compatibility. By the above lemma, $S$ satisfies the stable property. Hence we only have to check for all $s \in R S_{1}(S), s$ is not dead-lock. Suppose by the contradiction, $s$ contains the receiving states $t_{1}, \ldots, t_{n}$. Then by the multiparty compatibility, there exists 1 -bounded execution $\varphi$ such that $s \stackrel{\varphi}{\rightarrow} \stackrel{\bar{t}_{1}}{\rightarrow} s^{\prime}$. Hence $s^{\prime} \stackrel{t_{1}}{\rightarrow} s^{\prime \prime}$ and $s^{\prime \prime}$ is stable. Applying this routine to the rest of receiving states $t_{2}, \ldots, t_{n}$, we conclude the proof.

\section{B.2 Proof for Lemma 4.1}

Proof. We prove by induction that $\forall n, S_{1} \approx_{n} S_{2} \Longrightarrow S_{1} \approx_{n+1} S_{2}$. Then the lemma follows.

We assume $S_{1} \approx_{n} S_{2}$ and then prove, by induction on the length of any execution $\varphi$ that uses less than $n$ buffer space in $S_{1}$, that $\varphi$ is accepted by $S_{2}$. If the length $|\varphi|<n+1$, 
then the buffer usage of $\varphi$ for $S_{1}$ cannot exceed $n$, therefore $S_{2}$ can realise $\varphi$ since $S_{1} \approx_{n} S_{2}$.

Assume that a trace $\varphi$ in $S_{1}$ has length $|\varphi|=k+1$, that $\varphi$ is $(n+1)$-bound, and that any trace strictly shorter than $\varphi$ or using less buffer space is accepted by $S_{2}$.

We denote the last action of $\varphi$ as $\ell$. We name $\ell_{0}$ the last unmatched send transition pq! $a$ of $\varphi$ that is not $\ell$. We can therefore write $\varphi$ as $\varphi_{0} \ell_{0} \varphi_{1} \ell$, with $\varphi_{1}$ minimal. I.e. there is no permutation such that $\varphi_{0} \ell \varphi_{0}^{\prime} \ell_{0}$. In $S_{1}$, we have

$$
S_{1}: s_{0} \stackrel{\varphi_{0}}{\longrightarrow} \stackrel{\ell_{0}}{\longrightarrow} \stackrel{\varphi_{1}}{\longrightarrow} s_{1} \stackrel{\ell}{\rightarrow} s
$$

By Lemma B.5, we have a trace $\varphi_{2}$ such that:

$$
S_{1}: s_{0} \stackrel{\varphi_{0}}{\longrightarrow} \stackrel{\ell_{0}}{\longrightarrow} \stackrel{\varphi_{1}}{\longrightarrow} s_{1} \stackrel{\varphi_{2}}{\longrightarrow} \stackrel{\overline{\ell_{0}}}{\longrightarrow} s_{1}^{\prime}
$$

Case $\varphi_{2}=\varepsilon$. Hence

$$
S_{1}: s_{0} \stackrel{\varphi_{0}}{\rightarrow} \stackrel{\ell_{0}}{\rightarrow} \stackrel{\varphi_{1}}{\rightarrow} s_{1} \stackrel{\overline{\ell_{0}}}{\rightarrow} s_{1}^{\prime} \quad \text { and } \quad s_{1} \stackrel{\ell}{\rightarrow} s
$$

Let $\ell=\mathrm{p}_{1} \mathrm{q}_{1} ! b$. Then by Lemma B.1 (3), $s_{1} \stackrel{\overline{\ell_{0}}}{\rightarrow} \stackrel{\ell}{\rightarrow} s^{\prime \prime}$ as required.

Case $\varphi_{2}=\ell_{1} \cdot \varphi_{2}^{\prime}$.

1. If $\ell=\mathrm{p}_{1} \mathrm{q}_{1} ! b$ and $\ell_{1}=\mathrm{p}_{2} \mathrm{q}_{2} ? c$, then by Lemma B.1 (3), $s_{1} \stackrel{\ell_{1}}{\rightarrow} \stackrel{\ell}{\rightarrow} s^{\prime \prime}$. Hence we apply the induction on $\varphi_{2}^{\prime}$

2. If $\ell=\mathrm{p}_{1} \mathrm{q}_{1} ! b$ and $\ell_{1}=\mathrm{p}_{2} \mathrm{q}_{2} ! c$, then by directedness, we have three cases:

(a) $\mathrm{p}_{1} \neq \mathrm{p}_{2}$ and $\mathrm{q}_{1} \neq \mathrm{q}_{2}$. By Lemma B.1 (1), we have

$$
s_{1} \stackrel{\ell_{2}}{\rightarrow} s \stackrel{\ell}{\rightarrow} s_{2}^{\prime} \stackrel{\varphi_{2}^{\prime}}{\rightarrow} s_{1}^{\prime}
$$

Hence we conclude by the induction on $\varphi_{2}^{\prime}$.

(b) $\mathrm{p}_{1}=\mathrm{p}_{2}$ and $\mathrm{q}_{1}=\mathrm{q}_{2}$ and $b \neq c$.

In this case, by Lemma B.5, there exists $\varphi_{3}$ such that $s_{1} \stackrel{\ell}{\rightarrow} \stackrel{\varphi_{3}}{\rightarrow} \stackrel{\overline{\ell_{0}}}{\rightarrow}$. Hence this case is subsumed into (a) or (c) below.

(c) $\mathrm{p}_{1}=\mathrm{p}_{2}$ and $\mathrm{q}_{1}=\mathrm{q}_{2}$ and $b=c$.

Since $\ell_{0}$ and $\ell$ is not permutable, there is the causality such that $t_{0} \triangleleft t_{1} \triangleleft \cdots \triangleleft t_{n} \triangleleft$ $\cdots \triangleleft t_{n+m}$ with $\operatorname{act}\left(t_{0}\right)=\ell_{0}, \operatorname{act}\left(t_{n}\right)=\ell$ and $\operatorname{act}\left(t_{n+m}\right)=\overline{\ell_{0}}$. We note that since $l_{0}$ is the first outstanding output, by multiparty compatibility, $t_{i}(1 \leq i \leq n-1)$ does not include $\mathrm{p}_{1} \mathrm{q}_{1}$ ?a. Then by Lemma B.3, this case does not exist.

Applying Case (a), we can build in $S_{1}$ a sequence of transitions that allows $\ell$ using strictly less buffer space as:

$$
S_{1}: s_{0} \stackrel{\varphi_{0}}{\longrightarrow} \stackrel{\varphi_{0}^{\prime}}{\longrightarrow} \stackrel{\ell_{0}}{\longrightarrow} \stackrel{\varphi_{3}}{\longrightarrow} \stackrel{\overline{\ell_{0}}}{\longrightarrow} \stackrel{\ell}{\rightarrow}
$$

where $\varphi_{3}$ is the result of the combination of $\varphi_{1}$ and $\varphi_{2}$ using commutation.

By the assumption $\left(S_{1} \approx_{n} S_{2}\right), S_{2}$ can simulate this sequence as:

$$
S_{2}: s_{0} \stackrel{\varphi_{0}}{\longrightarrow} \stackrel{\varphi_{0}^{\prime}}{\longrightarrow} \stackrel{\ell_{0}}{\longrightarrow} \stackrel{\varphi_{3}}{\longrightarrow} \stackrel{\overline{\ell_{0}}}{\longrightarrow} \stackrel{\ell}{\rightarrow}
$$




$$
\begin{aligned}
\mathbf{G}=\operatorname{def} \mathbf{x}_{0} & =\mathbf{x}_{1} \mid \mathbf{x}_{2} \\
\mathbf{x}_{1}+\mathbf{x}_{5} & =\mathbf{x}_{3} \\
\mathbf{x}_{3} & =\mathrm{A} \rightarrow \mathrm{B}: \text { data } ; \mathbf{x}_{4} \\
\mathbf{x}_{4} & =\mathbf{x}_{5}+\mathbf{x}_{6} \\
\mathbf{x}_{6} & =\mathrm{A} \rightarrow \mathrm{B}: \text { eof } ; \mathbf{x}_{7} \\
\mathbf{x}_{2} & =\mathrm{A} \rightarrow \mathrm{C}: \log ; \mathbf{x}_{8} \\
\mathbf{x}_{7} \mid \mathbf{x}_{8} & =\mathbf{x}_{9} \\
\mathbf{x}_{9} & =\mathrm{B} \rightarrow \mathrm{C}: \text { save } ; \mathbf{x}_{10} \\
\mathbf{x}_{10} & =\text { end in } \mathbf{x}_{0}
\end{aligned}
$$

Data transfer example

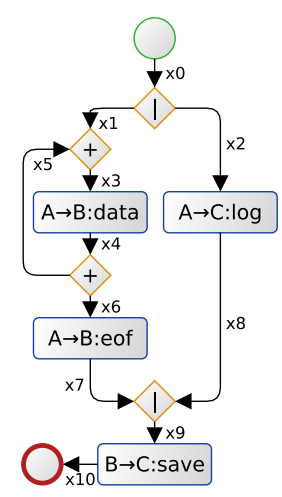

Fig. 3. Generalised global type and graph representation

All the commutation steps used in $S_{1}$ are also valid in $S_{2}$ since they are solely based on causalities of the transition sequences. We therefore can permute (B.7) back to:

$$
S_{2}: s_{0} \stackrel{\varphi_{0}}{\longrightarrow} \stackrel{\ell_{0}}{\longrightarrow} \stackrel{\varphi_{3}}{\longrightarrow} \stackrel{\ell}{\rightarrow}
$$

It concludes this proof.

\section{Generalised Multiparty Session Automata}

As an addition to the main results, we extend the results obtained on classical multiparty session types to tackle generalised multiparty session types [11], an extension with new features such as flexible fork, choice, merge and join operations for precise flow specification. It strictly subsumes classical MPST.

\section{C.1 Generalised global and local types}

In this subsection, we recall definitions from [11].

Generalised global types We first define generalised global types. The syntax is defined below.

$$
\begin{aligned}
& \text { G }::=\operatorname{def} \widetilde{G} \text { in } \mathbf{x} \quad \text { Global type }
\end{aligned}
$$

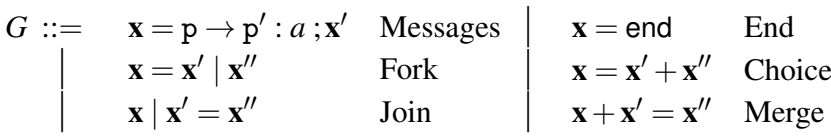

A global type $\mathbf{G}=\operatorname{def} \widetilde{G}$ in $\mathbf{x}_{0}$ describes an interaction between a fixed number of participants. We explain each of the constructs by example, in Figure 3, alongside the corresponding graphical representation inspired by the BPMN 2.0 business processing language. This example features three participants, with $A$ sending data to $B$ while $C$ concurrently records a log entry of the transmission.

The prescribed interaction starts from $\mathbf{x}_{0}$, which we call the initial state (in green in the graphical representation), and proceeds according to the transitions specified in $\widetilde{G}$ (the diamond or boxes operators in the picture). The state variables $\mathbf{x}$ in $\widetilde{G}$ (the edges in the graph) represent the successive distributed states of the interaction. Transitions can be message exchanges of the form $\mathbf{x}_{3}=\mathrm{A} \rightarrow \mathrm{B}:$ data $; \mathbf{x}_{4}$ where this transition specifies that $A$ can go from $\mathbf{x}_{3}$ to the continuation $\mathbf{x}_{4}$ by sending message data, while $B$ goes from $\mathbf{x}_{3}$ to $\mathbf{x}_{4}$ by receiving it. In the graph, message exchanges are represented 
by boxes with exactly one incoming and one outgoing edges. $\mathbf{x}_{4}=\mathbf{x}_{5}+\mathbf{x}_{6}$ represents the choice between continuing with $\mathbf{x}_{5}$ or $\mathbf{x}_{6}$ and $\mathbf{x}_{0}=\mathbf{x}_{1} \mid \mathbf{x}_{2}$ represents forking the interactions, allowing the interleaving of actions at $\mathbf{x}_{1}$ and $\mathbf{x}_{2}$. These forking threads are eventually collected by joining construct of the form $\mathbf{x}_{7} \mid \mathbf{x}_{8}=\mathbf{x}_{9}$. Similarly choices (i.e. mutually exclusive paths) are closed by merging construct $\mathbf{x}_{1}+\mathbf{x}_{5}=\mathbf{x}_{3}$, where they share a continuation. Forks, choices, joins and merges are represented by diamond ternary operators in the graphical notation. Fork and choice have one input and two outputs, join and merge have two inputs and one output. Fork and join use the diamond operator with the $\mid$ symbol, while choice and merge use a diamond with the + symbol. The $\mathbf{x}_{10}=$ end transition is represented by a red circle. Note that the two representations (syntax and graph) are equivalent.

The motivation behind this choice of syntax is to support general control flows, as classical global type syntax tree, even with added operators fork $\mid$ and choice $+[3,7$, $10,17]$, is limited to series-parallel control flow graphs.

Generalised local types As for global types, a local type $\mathbf{T}$ follows a shape of a state machine-like definition: local types are of the form def $\widetilde{T}$ in $\mathbf{x}_{0}$. The different actions include send ( $\mathrm{p} ! a$ is the action of sending to $\mathrm{p}$ a message $a$ ), receive ( $\mathrm{p}$ ? $a$ is the action of receiving from $\mathrm{p}$ a message $a$ ), fork, internal choice, external choice, join, merge, indirection and end. Note that merge is used for both internal and external choices. Similarly to global types, an obvious graphical representation exists.

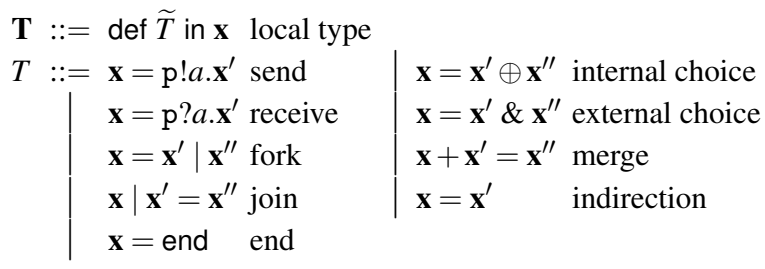

The local types are obtained from the global type by successive projection to each participant. We define the projection of a well-formed global type $\mathbf{G}$ to the local type of participant $\mathrm{p}$ (written $\mathbf{G} \uparrow \mathrm{p}$ ). The projection is given in Appendix D because it is straightforward: for example, $\mathbf{x}=\mathrm{p} \rightarrow \mathrm{q}: a ; \mathbf{x}^{\prime}$ is projected to the output $\mathbf{x}=\mathrm{p}^{\prime} ! a \cdot \mathbf{x}^{\prime}$ from p's viewpoint and an input $\mathbf{x}=\mathrm{p} ? a \cdot \mathbf{x}^{\prime}$ from q's viewpoint; otherwise it creates an indirection link from $\mathbf{x}$ to $\mathbf{x}^{\prime}$. Choice $\mathbf{x}=\mathbf{x}^{\prime}+\mathbf{x}^{\prime \prime}$ is projected to the internal choice $\mathbf{x}=\mathbf{x}^{\prime} \oplus \mathbf{x}^{\prime \prime}$ if $\mathrm{p}$ is the unique participant deciding on which branch to choose; otherwise the projection gives an external choice $\mathbf{x}=\mathbf{x}^{\prime} \& \mathbf{x}^{\prime \prime}$ ([11] gives the definition). Forks, joins and merges are kept identical. As an example, Figure 6 features on the left, in graphical notation, the result of the projection to $A$ from the global type $\mathbf{G}$ of Figure 3. Its structure is exactly the same as the original global type, except for the silent transition $\mathbf{x}_{9}=\mathbf{x}_{10}$ which is silent from the point of view of $A$ and therefore is just elided in the local type.

\section{C.2 Labelled transitions of generalised global and local types}

It is possible to define a labelled semantics for global and local types by considering the type (whether local or global) as a state machine specification in which each participant (or the participant, in the case of local type) can evolve, as they would in a CFSMs. As for CFSMs and classical multiparty session types, we keep the syntax of labels $\left(\ell, \ell^{\prime}, \ldots\right)$. 


$$
\begin{aligned}
& \frac{\mathbf{x}=\mathrm{p} \rightarrow \mathrm{p}^{\prime}: a ; \mathbf{x}^{\prime} \in \widetilde{G} \quad \mathbf{X}_{\mathrm{p}}=\mathbf{X}[\mathbf{x}] \quad w_{\mathrm{pp}^{\prime}} \in \tilde{w}}{\operatorname{def} \widetilde{G} \text { in } \tilde{\mathbf{X}}, \tilde{w} \stackrel{\mathrm{pp}^{\prime} ! a}{\longrightarrow} \operatorname{def} \widetilde{G} \text { in } \tilde{\mathbf{X}}\left[\mathbf{X}_{\mathrm{p}} \leftarrow \mathbf{X}\left[\mathbf{x}^{\prime}\right]\right], \tilde{w}\left[w_{\mathrm{pp}^{\prime}} \leftarrow w_{\mathrm{pp}^{\prime}} \cdot a\right]}\lfloor\text { GGR } 1\rfloor
\end{aligned}
$$

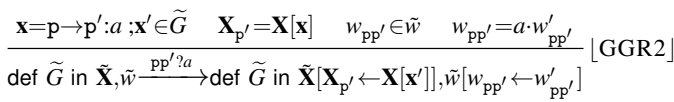

$$
\begin{aligned}
& \mathbf{x}=\mathrm{p} \rightarrow \mathrm{p}^{\prime}: a ; \mathbf{x}^{\prime} \in \widetilde{G} \quad \mathbf{X}_{\mathrm{q}}=\mathbf{X}[\mathbf{x}] \quad \mathrm{q} \notin\left\{\mathrm{p}, \mathrm{p}^{\prime}\right\} \\
& \frac{\operatorname{def} \widetilde{G} \text { in } \tilde{\mathbf{X}}\left[\mathbf{X}_{\mathrm{q}} \leftarrow \mathbf{X}\left[\mathbf{x}^{\prime}\right]\right], \tilde{w} \stackrel{\ell}{\rightarrow} \operatorname{def} \widetilde{G} \text { in } \tilde{\mathbf{X}}^{\prime}, \tilde{w}^{\prime}}{\operatorname{def} \widetilde{G} \text { in } \tilde{\mathbf{X}}, \tilde{w} \stackrel{\ell}{\rightarrow} \operatorname{def} \widetilde{G} \text { in } \tilde{\mathbf{X}}^{\prime}, \tilde{w}^{\prime}}\lfloor\text { GGR3 } \\
& \frac{\mathbf{X}_{\mathrm{p}}=\mathbf{X} \quad \mathbf{X} \equiv \equiv_{\tilde{G}} \mathbf{X}^{\prime} \quad \operatorname{def} \widetilde{G} \text { in } \tilde{\mathbf{X}}\left[\mathbf{X}_{\mathrm{p}} \leftarrow \mathbf{X}^{\prime}\right], \tilde{w} \stackrel{\ell}{\rightarrow} \operatorname{def} \widetilde{G} \text { in } \tilde{\mathbf{X}}^{\prime}, \tilde{w}^{\prime}}{\operatorname{def} \widetilde{G} \text { in } \tilde{\mathbf{X}}, \tilde{w} \stackrel{\ell}{\rightarrow} \operatorname{def} \widetilde{G} \text { in } \tilde{\mathbf{X}}^{\prime}, \tilde{w}^{\prime}}\lfloor\text { GGR } 4\rfloor
\end{aligned}
$$

Fig. 4. Global LTS

We use the following notation to keep track of local states (with parallelism, each participant can now execute several transitions concurrently):

$$
\mathrm{X}::=\mathbf{x}_{i}|\mathrm{X}| \mathrm{X} \quad \mathrm{X}[-]::={ }_{-}|\mathrm{X}[-]| \mathrm{X}|\mathrm{X}| \mathrm{X}[-]
$$

LTS for global types We first define, for a global type $\mathbf{G}=\operatorname{def} \widetilde{G}$ in $\mathbf{x}_{0}$, a transition system def $\widetilde{G}$ in $\tilde{\mathbf{X}}, \tilde{w} \stackrel{\ell}{\rightarrow}$ def $\widetilde{G}$ in $\tilde{\mathbf{X}}^{\prime}, \tilde{w}^{\prime}$, where $\tilde{\mathbf{X}}$ and $\tilde{\mathbf{X}}^{\prime}$ represents a vector recording the state of each of the participants $\tilde{\mathbf{X}}=\left\{\mathbf{X}_{\mathrm{p}}\right\}_{\mathrm{p} \in \mathcal{P}}$ and where $\tilde{w}$ represents the content of the

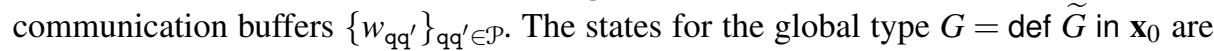
equipped with an equivalence relation $\equiv_{\widetilde{G}}$, defined in Appendix D.1, which covers associativity, commutativity, forks and joins, choices and merges. Initially, $\tilde{\mathbf{X}}_{0}=\left\{\mathbf{x}_{0}\right\}_{\mathrm{p} \in \mathcal{P}}$

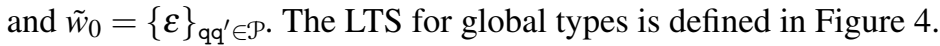

The semantics of global types, as given by the rules $\lfloor$ GGR 1,2$\rfloor$, follows the intuition of communicating systems: if the global type allows, a participant at the right state can

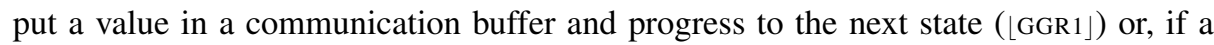

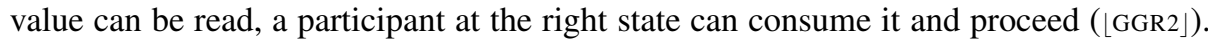
Rule [GGR3」 allows participants that are not concerned by a transition to go there for free. Fork, join, choice and merge transitions are passed through silently by rule [GGR4].

LTS for local types We define in Figure 5 a transition system $\widetilde{\mathbf{T}}, \tilde{w} \stackrel{\ell}{\rightarrow} \widetilde{\mathbf{T}}^{\prime}, \tilde{w}^{\prime}$, where $\widetilde{\mathbf{T}}$ represents a set of local types $\left\{\text { def } \widetilde{T} \text { in } \tilde{\mathbf{X}}_{\mathrm{p}}\right\}_{\mathrm{p} \in \mathcal{P}}$ and $\tilde{w}$ represents the content of

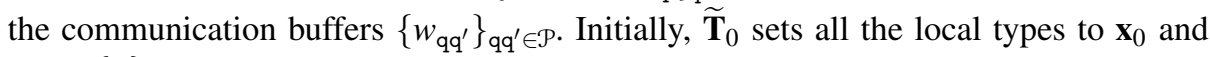
$\tilde{w}_{0}=\{\varepsilon\}_{\mathrm{qq}^{\prime} \in \mathcal{P}}$. The principle is strictly identical to the LTS for global types, with, again, an omitted structural equivalence $\equiv_{\widetilde{T}}$ between local states.

Equivalence between generalised local and global types Given the similarity in principle between the global and local LTSs, and considering that the projection algorithm for generalised global types is quasi-homomorphic, we can easily get the trace equivalence between the local and global semantics.

Theorem C.1 (soundness and completeness of projection). If $\overrightarrow{\mathbf{T}}$ is the projection of a global type $\mathbf{G}$ to all roles, then $\mathbf{G} \approx(\overrightarrow{\mathbf{T}}, \varepsilon)$.

\section{C.3 Translations between general local types and CFSMs}

Now that we have proved the equivalence from global to local types, we establish the conversion of local types to and from CFSMs. 


$$
\begin{aligned}
& \frac{\mathbf{x}=\mathrm{p}^{\prime} ! a \cdot \mathbf{x}^{\prime} \in \widetilde{T}}{\widetilde{\mathbf{T}}, \tilde{w} \stackrel{\mathrm{pp}_{\mathrm{p}} ! a}{\longrightarrow} \widetilde{\mathbf{T}}\left[\mathbf{T}_{\mathrm{p}} \leftarrow \operatorname{def} \widetilde{T} \widetilde{T} \text { in } \mathrm{X}\left[\mathbf{x}\left[\mathbf{x}^{\prime}\right]\right], \widetilde{w}\left[w_{\mathrm{pp}^{\prime}} \leftarrow w_{\mathrm{pp}^{\prime}} \cdot w_{\mathrm{pp}^{\prime}} \in \tilde{w} ! a\right]\right.}\lfloor\text { GLR } 1\rfloor
\end{aligned}
$$

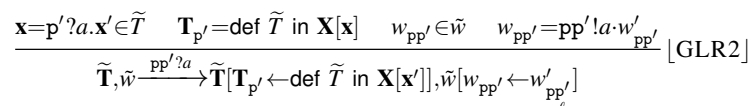

$$
\begin{aligned}
& \frac{\mathbf{T}_{\mathrm{p}}=\operatorname{def} \widetilde{T} \text { in } \mathbf{X} \quad \mathbf{X} \equiv \equiv_{\tilde{T}} \mathbf{X}^{\prime} \quad \widetilde{T}\left[\mathbf{T}_{\mathrm{p}} \leftarrow \operatorname{def} \widetilde{T} \text { in } \mathbf{X}^{\prime}\right], \tilde{w} \stackrel{\ell}{\rightarrow} \widetilde{\mathbf{T}}^{\prime}, \tilde{w}^{\prime}}{\widetilde{T}, \tilde{w} \stackrel{\ell}{\rightarrow} \widetilde{T}^{\prime}, \tilde{w}^{\prime}}\lfloor\text { GLR3 }\rfloor
\end{aligned}
$$

Fig. 5. Local LTS

Translation to CFSMs We first give the already known translation from local types to CFSMs [11]. The illustration of that translation on the Data transfer example is given on the top-right corner of Figure 6.

Definition C.1 (translation from local types to CFSMs [11]). If $\mathbf{T}=\operatorname{def} \widetilde{T}$ in $\mathbf{x}_{0}$ is the local type of participant $\mathrm{p}$ projected from $\mathbf{G}$, then the corresponding automaton is $\mathcal{A}(\mathbf{T})=\left(Q, C, q_{0}, \mathbb{A}, \delta\right)$ where:

- $Q$ is defined as the set of well-formed states $\mathrm{X}$ built from the state variables $\left\{\mathbf{x}_{i}\right\}$ of

T. $Q$ is defined up to the equivalence relation $\equiv_{\widetilde{T}}$ mentioned in $\S$ C.2.

- $C=\{\mathrm{pq} \mid \mathrm{p}, \mathrm{q} \in \mathbf{G}\}$

$-q_{0}=\mathbf{x}_{0}$

$-\Sigma$ is the set of $\{a \in \mathbf{G}\}$

- $\delta$ is defined by:

- $\left(\mathrm{X}[\mathbf{x}],\left(\mathrm{pp}^{\prime} ! a\right), \mathrm{X}\left[\mathbf{x}^{\prime}\right]\right) \in \delta$ if $\mathbf{x}=\mathrm{p}^{\prime} ! a \cdot \mathbf{x}^{\prime} \in \widetilde{T}$.

- $\left(\mathrm{X}[\mathbf{x}],\left(\mathrm{p}^{\prime} \mathrm{p} ? a\right), \mathrm{X}\left[\mathbf{x}^{\prime}\right]\right) \in \delta$ if $\mathbf{x}=\mathrm{p}^{\prime} ? a \cdot \mathbf{x}^{\prime} \in \widetilde{T}$.

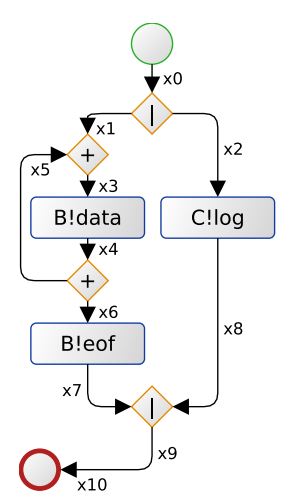

General local type for A
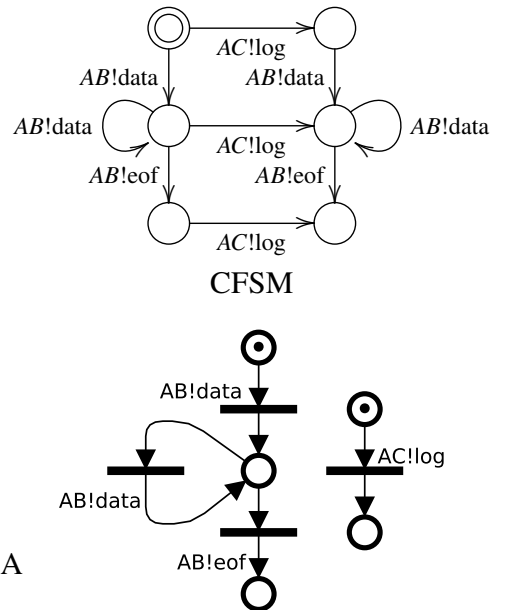

Inferred labelled Petri net

Fig. 6. Data transfer example: local translations

Translations from CFSMs The converse translation is not as obvious as local types feature explicit forks and joins, while CFSMs only propose choices between interleaved sequences. The translation from a CFSM to a local type therefore comes in 3 steps. 
First, we apply a generic translation from minimised CFSMs to Petri nets [9,21]. This translation relies on the polynomial computation of the graph of regions [1], preserves the trace semantics of the CFSM and, by the minimality of the produced net, makes the concurrency explicit. Figure 6 illustrates on the Data transfer example the shape of the Petri net that can be produced by such a generic translation. Note that the produced Petri net is always safe and free choice.

The second step of the conversion is to take the Petri net with labelled transitions and enrich it with new silent transitions and new places so that it can be translated into local types. Notably, it should have only one initial marked place, one final place and all labelled transitions should have exactly one incoming and one outgoing arc. Then, we constrain all transitions to be linked with no more than 3 arcs ( 2 incoming and 1 outgoing for a join transition, or 1 incoming and 2 outgoing for a fork transition, 1 incoming and 1 outgoing for all the other transitions). Places should have no more that 2 incoming and 2 outgoing arcs: if there are two incoming (merge), then the transitions they come from should only have one incoming arc each; if there are 2 outgoing (choice), then the transitions they lead to should have only one outgoing arc each.

In the end, the translation to local type is simple, as each place corresponds to a state variable $\mathbf{x}$, and the different local type transitions can be simply identified. For the lightness of the presentation, instead of defining formally this last step, we describe the converse translation. From it, it is possible to infer the local type generation.

Definition C.2 (Petri net representation). Given a local type $\mathbf{T}=\operatorname{def} \widetilde{T}$ in $\mathbf{x}_{0}$, we define the Petri net $\mathbb{P}(\mathbf{T})$ by:

- Each state variable $\mathbf{x} \in \widetilde{T}$ is a place in $\mathbb{P}(\mathbf{T})$.

- All the places are initially empty, except for one token in $\mathbf{x}_{0}$.

- Transitions in $\widetilde{T}$ are translated as follows:

- If $\mathbf{x}=\mathrm{p} ! a \cdot \mathbf{x}^{\prime} \in \widetilde{T}$ then there is a transition labelled in $\mathbb{P}(\mathbf{T})$, whose unique input $\operatorname{arc}$ comes from $\mathbf{x}$ and whose unique output arc goes to $\mathbf{x}^{\prime}$.

- If $\mathbf{x}=\mathrm{p} ? a \cdot \mathbf{x}^{\prime} \in \widetilde{T}$ then their is a transition in $\mathbb{P}(\mathbf{T})$, whose unique input arc comes from $\mathbf{x}$ and whose unique output arc goes to $\mathbf{x}^{\prime}$.

- If $\mathbf{x}_{1}=\mathbf{x}_{2} \mid \mathbf{x}_{3} \in \widetilde{T}$ then there is a transition in $\mathbb{P}(\mathbf{T})$, whose unique input arc comes from $\mathbf{x}_{1}$ and whose two outputs arcs go to $\mathbf{x}_{2}$ and $\mathbf{x}_{3}$.

- If $\mathbf{x}_{1}=\mathbf{x}_{2}+\mathbf{x}_{3} \in \widetilde{T}$ (internal or external choice) then there are two transitions in $\mathbb{P}(\mathbf{T})$, that each have an input arc from $\mathbf{x}_{1}$ and that respectively have an output arc to $\mathbf{x}_{2}$ and $\mathbf{x}_{3}$.

- If $\mathbf{x}_{1}+\mathbf{x}_{2}=\mathbf{x}_{3} \in \widetilde{T}$ then there are two transitions in $\mathbb{P}(\mathbf{T})$, that respectively have an input arc from $\mathbf{x}_{1}$ and $\mathbf{x}_{2}$ and that both have an output arc to $\mathbf{x}_{3}$.

- If $\mathbf{x}_{1} \mid \mathbf{x}_{2}=\mathbf{x}_{3} \in \widetilde{T}$ then there is a transition in $\mathbb{P}(\mathbf{T})$, whose two input arcs respectively come from $\mathbf{x}_{1}$ and $\mathbf{x}_{2}$ and whose unique output arc goes to $\mathbf{x}_{3}$.

The idea of the translation back from a Petri net to a local type is to identify the transitions and place patterns and convert them into local type transitions.

Note that, in Figure 6, the inferred Petri Net will not give back the local type on the left: in the general case, going through the translation from local type to CFSM and then back to local type will only give an isomorphic local type. The traces are of course preserved. 


\section{C.4 Parallelism and local choice condition}

This subsection introduces the conditions that CFSMs should respect in order to correspond to well-formed local types projected from generalised global types. It extends the conditions that were sufficient for classical multiparty session types for two reasons. First, we now have concurrent interactions and the no-mixed choice condition does not hold anymore. Second, the well-formedness condition corresponding to projectability in classical multiparty session types needs to take into account the complex control flows of generalised multiparty session types.

We start by a commutativity condition for mixed states in CFSMs: a state is mixed parallel if any send transition satisfies the diamond property with any receive transition. Formally:

Definition C.3 (mixed parallel). Let $M=\left(Q, C, q_{0}, \mathbb{A}, \delta\right)$. We say local state $q$ in $M$ is mixed parallel if for all $\left(q, \ell_{1}, q_{1}^{\prime}\right),\left(q, \ell_{2}, q_{2}^{\prime}\right) \in \delta$ such that $\ell_{1}$ is a send and $\ell_{2}$ is a receive we have $\left(q_{1}^{\prime}, \ell_{2}, q^{\prime}\right),\left(q_{2}^{\prime}, \ell_{1}, q^{\prime}\right) \in \delta$ for some $q^{\prime}$.

Next, we introduce two conditions for the choice that are akin to the local choice conditions with additional data of [13, Def. 2] or the "knowledge of choice" conditions of [7].

\section{Definition C.4 (local choice condition).}

1. The set of receivers of transitions $s_{1} \frac{t_{1} \cdots t_{m}}{s_{m+1}}$ is defined as $\operatorname{Rcv}\left(t_{1} \cdots t_{m}\right)=\{\mathrm{q} \mid$ $\left.\exists i \leq m, t_{i}=\left(s_{i}, \mathrm{pq} ? a, s_{i+1}\right)\right\}$

2. The set of active senders are defined as $A \operatorname{Send}\left(t_{1} \cdots t_{m}\right)=\left\{\mathrm{p} \mid \exists i \leq m, t_{i}=\left(s_{i}, \mathrm{pq} ! a, s_{i+1}\right) \wedge\right.$ $\left.\forall k<i . t_{k} \neq\left(s_{k}, \mathrm{p}^{\prime} \mathrm{p} ? b, s_{k+1}\right)\right\}$ and represent the participants who could immediately send from state $s_{1}$.

3. Suppose $s_{0} \varphi_{\rightarrow}$ and $\varphi=\varphi_{0} \cdot t_{1} \cdot \varphi_{1} \cdot t_{2} \cdot \varphi_{2}$. We write $t_{1} \triangleleft t_{2}\left(t_{2}\right.$ depends on $\left.t_{1}\right)$ if either (1) $\Phi\left(\operatorname{act}\left(t_{2}\right)\right)=\operatorname{act}\left(t_{1}\right)$ or (2) $\operatorname{subj}\left(t_{1}\right)=\operatorname{subj}\left(t_{2}\right)$ unless $t_{1}$ and $t_{2}$ are parallel.

4. We say $\varphi=t_{0} \cdot t_{1} \cdot t_{2} \cdots t_{n}$ is the causal chain if $s_{0} \stackrel{\varphi^{\prime}}{\rightarrow} s^{\prime}$ and $\varphi \subseteq \varphi^{\prime}$ with, for all $0 \leq k \leq n-1$, there exists $i$ such that $i>k$ and $t_{k} \triangleleft t_{i}$.

5. $S$ satisfies the receiver property if, for all $s \in R S(S)$ and $s \stackrel{t_{1}}{\rightarrow} s_{1}$ and $s \stackrel{t_{2}}{\rightarrow} s_{2}$ with $a c t\left(t_{i}\right)=\mathrm{pq}_{i} ! a_{i}$, there exist $s_{1} \stackrel{\varphi_{1}}{\longrightarrow} s_{1}^{\prime}$ and $s_{2} \stackrel{\varphi_{2}}{\rightarrow} s_{2}^{\prime}$ such that $\operatorname{Rcv}\left(\varphi_{1}\right)=\operatorname{Rcv}\left(\varphi_{2}\right)$.

6. $S$ satisfies the unique sender property if $s_{0} \stackrel{\varphi_{1}}{\longrightarrow} s_{1} \stackrel{t_{1}}{\rightarrow} s_{1}^{\prime}$ and $s_{0} \stackrel{\varphi_{2}}{\longrightarrow} s_{2} \stackrel{t_{2}}{\longrightarrow} s_{2}^{\prime}$, with $\operatorname{act}\left(t_{1}\right)=$ $\mathrm{p}_{1} \mathrm{p} ? a_{1}, \operatorname{act}\left(t_{2}\right)=\mathrm{p}_{2} \mathrm{p} ? a_{2}, a_{1} \neq a_{2}, \neg t_{1} \triangleleft t_{2}$ and $\neg t_{2} \triangleleft t_{1}$, and $\varphi_{i} \cdot t_{i}$ the maximum causal chain. Then $A \operatorname{Send}\left(\varphi_{1} \cdot t_{1}\right)=\operatorname{ASend}\left(\varphi_{2} \cdot t_{2}\right)=\{\mathrm{q}\}$.

Together with multiparty compatibility, the receiver property ensures deadlock-freedom while the unique sender property guarantees orphan message-freedom.

Proposition C.1 (stability). Suppose $S=\left\{M_{\mathrm{p}}\right\}_{\mathrm{p} \in \mathcal{P}}$ and each $M_{\mathrm{p}}$ is deterministic. If (1) $S$ is multiparty compatible; (2) each mixed state in $S$ is mixed parallel; and (3) for any local state that can do two receive transitions, either they commute (satisfy the diamond property) or the state satisfies the unique sender condition, then $S$ is stable and satisfies the reception error freedom and orphan message-freedom properties.

Proof. The proof is similar to Proposition 4.1, noting that the unique sender condition guarantees the input availability. See Appendix D. 
Theorem C.2 (deadlock-freedom). Suppose $S=\left\{M_{\mathrm{p}}\right\}_{\mathrm{p} \in \mathcal{P}}$ satisfies the same conditions as Proposition C.1. Assume, in addition, that $S$ satisfies the receiver condition. Then $S$ is deadlock-free.

Proof. We deduce this theorem from the stability property and the receiver condition. The proof uses a similar reasoning as Proposition 4.1.

We call the systems that satisfy the conditions of Theorem C.2 session-compatible.

By the same algorithm, the multiparty compatibility property is decidable for systems of deterministic CFSMs. It is however undecidable to check the receiver and unique sender properties in general. On the other hand, once multiparty compatibility is assumed, we can restrict the checks to 1-bounded executions (i.e. we limit $\varphi_{1}, \varphi_{2}$, $\varphi_{1}^{\prime}$ and $\varphi_{2}^{\prime}$ to 1-bounded executions and $R S_{1}(S)$ in Definition C.4). Then these properties become decidable. Combining the synthesis algorithm defined below, we can decide a subset of CFSMs which can build a general, well-formed global type.

\section{C.5 Synthesis of general multiparty session automata}

Now all the pieces are in place for the main results of this paper. We are able to identify the class of communicating systems that correspond to generalised multiparty session types.

The main theorems in this section follow:

Theorem C.3 (synthesis of general systems). Suppose $S=\left\{M_{\mathrm{p}}\right\}_{\mathrm{p} \in \mathcal{P}}$ is a sessioncompatible system. Then there is an algorithm which builds $\mathbf{G}$ such that $S \approx \mathbf{G}$.

Proof. The algorithms is the following. We consider $S=\left\{M_{\mathrm{p}}\right\}_{\mathrm{p} \in \mathcal{P}}$ as the definition of a transition system. In this transition system, we only consider the 1-bounded executions. This restriction produces a finite state LTS, where send transitions are immediately followed by the unique corresponding receive transition. In each of these cases, we replace the pair of transitions $\mathrm{pp}^{\prime} ! a$ and $\mathrm{pp}^{\prime} ? a$ by a unique transition $\mathrm{p} \rightarrow \mathrm{p}^{\prime}: a$. To obtain the global type $\mathbf{G}$, we then follow first the standard conversion to Petri nets and the equivalence between Petri nets and global types (similar to the one between Petri nets and local types). We conclude the equivalence by a version of Lemma 4.1 adapted to session-compatible system.

Using the synthesis theorem, we are able to provide a full characterisation of generalised multiparty session types in term of session-compatible systems.

Theorem C.4 (soundness and completeness in MSA). Suppose $S=\left\{M_{\mathrm{p}}\right\}_{\mathrm{p} \in \mathcal{P}}$ is a session compatible system. Then there exits $\mathbf{G}$ such that $S \approx \mathbf{G}$. Conversely, if $\mathbf{G}$ is wellformed as in [11], then there exits $S$ which satisfies the safety and liveness properties (deadlock-freedom, reception error-freedom and orphan message-freedom), and $S \approx \mathbf{G}$.

Proof. By Theorem C.3 and Theorem C.1 with the same reasoning as in Theorem 4.3. 


\section{Appendix for Section C}

Projection We define the projection from a global type to a local type where ASend means that a set of active senders, which corresponds to the same definition in CFSMs (see [11]).

$$
\begin{aligned}
& \operatorname{def} \widetilde{G} \text { in } \mathbf{x} \uparrow \mathrm{p}=\operatorname{def} \widetilde{G} \uparrow_{\widetilde{G}} \mathrm{p} \text { in } \mathbf{x} \\
& \mathbf{x}=\mathrm{p} \rightarrow \mathrm{p}^{\prime}: a ; \mathbf{x}^{\prime} \uparrow_{\widetilde{G}} \mathrm{p}=\mathbf{x}=\mathrm{p}^{\prime} ! a \cdot \mathbf{x}^{\prime} \\
& \mathbf{x}=\mathrm{p} \rightarrow \mathrm{p}^{\prime}: a ; \mathbf{x}^{\prime} \uparrow_{\widetilde{G}} \mathrm{p}^{\prime}=\mathbf{x}=\mathrm{p} ? a \cdot \mathbf{x}^{\prime} \\
& \mathbf{x}=\mathrm{p} \rightarrow \mathrm{p}^{\prime}: a ; \mathbf{x}^{\prime} \uparrow_{\widetilde{G}} \mathrm{p}^{\prime \prime}=\mathbf{x}=\mathbf{x}^{\prime}\left(\mathrm{p} \notin\left\{\mathrm{p}, \mathrm{p}^{\prime}\right\}\right) \\
& \mathbf{x}\left|\mathbf{x}^{\prime}=\mathbf{x}^{\prime \prime} \uparrow_{\widetilde{G}} \mathrm{p}=\mathbf{x}\right| \mathbf{x}^{\prime}=\mathbf{x}^{\prime \prime} \\
& \mathbf{x}=\mathbf{x}^{\prime}\left|\mathbf{x}^{\prime \prime} \uparrow_{\widetilde{G}} \mathrm{p}=\mathbf{x}=\mathbf{x}^{\prime}\right| \mathbf{x}^{\prime \prime} \\
& \mathbf{x}=\mathbf{x}^{\prime}+\mathbf{x}^{\prime \prime} \uparrow_{\widetilde{G}} \mathrm{p}=\mathbf{x}=\mathbf{x}^{\prime} \oplus \mathbf{x}^{\prime \prime} \quad(\text { if } \mathrm{p}=\operatorname{ASend}(\widetilde{G})(\mathbf{x})) \\
& \mathbf{x}=\mathbf{x}^{\prime}+\mathbf{x}^{\prime \prime} \uparrow_{\widetilde{G}} \mathrm{p}=\mathbf{x}=\mathbf{x}^{\prime} \& \mathbf{x}^{\prime \prime} \quad \text { (otherwise) } \\
& \mathbf{x}+\mathbf{x}^{\prime}=\mathbf{x}^{\prime \prime} \uparrow_{\widetilde{G}} \mathrm{p}=\mathbf{x}+\mathbf{x}^{\prime}=\mathbf{x}^{\prime \prime} \\
& \mathbf{x}=\text { end } \uparrow_{\widetilde{G}} \mathrm{p}=\mathbf{x}=\text { end }
\end{aligned}
$$

\section{D.1 Global type equivalence}

Below we define the equivalence relation $\equiv_{\widetilde{G}}$ used in the LTS of the global types.

$$
\begin{gathered}
\mathrm{X}\left|\mathrm{X}^{\prime} \equiv \widetilde{G} \mathrm{X}^{\prime}\right| \mathrm{X} \quad \mathrm{X}\left|\left(\mathrm{X}^{\prime} \mid \mathrm{X}^{\prime \prime}\right) \equiv \widetilde{G}\left(\mathrm{X} \mid \mathrm{X}^{\prime}\right)\right| \mathrm{X}^{\prime \prime} \\
\frac{\mathbf{x}=\mathbf{x}^{\prime} \in \widetilde{G}}{\mathrm{X}[\mathbf{x}] \equiv \widetilde{G} \mathrm{X}\left[\mathbf{x}^{\prime}\right]} \quad \frac{\mathbf{x}=\mathbf{x}^{\prime} \mid \mathbf{x}^{\prime \prime} \in \widetilde{G}}{\mathrm{X}[\mathbf{x}] \equiv \widetilde{G} \mathrm{X}\left[\mathbf{x}^{\prime} \mid \mathbf{x}^{\prime \prime}\right]} \quad \frac{\mathbf{x} \mid \mathbf{x}^{\prime}=\mathbf{x}^{\prime \prime} \in \widetilde{G}}{\mathrm{X}\left[\mathbf{x} \mid \mathbf{x}^{\prime}\right] \equiv \widetilde{G} \mathrm{X}\left[\mathbf{x}^{\prime \prime}\right]} \\
\frac{\mathbf{x}=\mathbf{x}^{\prime}+\mathbf{x}^{\prime \prime} \in \widetilde{G}}{\mathrm{X}[\mathbf{x}] \equiv \widetilde{G} \mathrm{X}\left[\mathbf{x}^{\prime}\right]} \quad \frac{\mathbf{x}=\mathbf{x}^{\prime}+\mathbf{x}^{\prime \prime} \in \widetilde{G}}{\mathrm{X}[\mathbf{x}] \equiv \widetilde{G}\left[\mathbf{x}^{\prime \prime}\right]} \quad \frac{\mathbf{x}+\mathbf{x}^{\prime}=\mathbf{x}^{\prime \prime} \in \widetilde{G}}{\mathrm{X}[\mathbf{x}] \equiv \widetilde{G} \mathrm{X}\left[\mathbf{x}^{\prime \prime}\right]} \quad \frac{\mathbf{x}+\mathbf{x}^{\prime}=\mathbf{x}^{\prime \prime} \in \widetilde{G}}{\mathrm{X}\left[\mathbf{x}^{\prime}\right] \equiv \widetilde{G} \mathrm{X}\left[\mathbf{x}^{\prime \prime}\right]}
\end{gathered}
$$

Below we define the equivalence relation $\equiv \widetilde{T}$ used in the translation in Definition C.1.

$$
\begin{aligned}
& \mathrm{X}\left|\mathrm{X}^{\prime} \equiv_{\widetilde{T}} \mathrm{X}^{\prime}\right| \mathrm{X} \quad \mathrm{X}\left|\left(\mathrm{X}^{\prime} \mid \mathrm{X}^{\prime \prime}\right) \equiv_{\widetilde{T}}\left(\mathrm{X} \mid \mathrm{X}^{\prime}\right)\right| \mathrm{X}^{\prime \prime} \\
& \frac{\mathbf{x}=\mathbf{x}^{\prime} \in \widetilde{T}}{\mathrm{X}[\mathbf{x}] \equiv_{\widetilde{T}} \mathrm{X}\left[\mathbf{x}^{\prime}\right]} \quad \frac{\mathbf{x}=\mathbf{x}^{\prime} \mid \mathbf{x}^{\prime \prime} \in \widetilde{T}}{\mathrm{X}[\mathbf{x}] \equiv_{\widetilde{T}} \mathrm{X}\left[\mathbf{x}^{\prime} \mid \mathbf{x}^{\prime \prime}\right]} \quad \frac{\mathbf{x} \mid \mathbf{x}^{\prime}=\mathbf{x}^{\prime \prime} \in \widetilde{T}}{\mathrm{X}\left[\mathbf{x} \mid \mathbf{x}^{\prime}\right] \equiv_{\widetilde{T}} \mathrm{X}\left[\mathbf{x}^{\prime \prime}\right]} \\
& \frac{\mathbf{x}=\mathbf{x}^{\prime} \& \mathbf{x}^{\prime \prime} \in \widetilde{T}}{\mathrm{X}[\mathbf{x}] \equiv_{\widetilde{T}} \mathrm{X}\left[\mathbf{x}^{\prime}\right]} \quad \frac{\mathbf{x}=\mathbf{x}^{\prime} \& \mathbf{x}^{\prime \prime} \in \widetilde{T}}{\mathrm{X}[\mathbf{x}] \equiv_{\widetilde{T}} \mathrm{X}\left[\mathbf{x}^{\prime \prime}\right]} \quad \frac{\mathbf{x}=\mathbf{x}^{\prime} \oplus \mathbf{x}^{\prime \prime} \in \widetilde{T}}{\mathrm{X}[\mathbf{x}] \equiv_{\widetilde{T}} \mathrm{X}\left[\mathbf{x}^{\prime}\right]} \quad \frac{\mathbf{x}=\mathbf{x}^{\prime} \oplus \mathbf{x}^{\prime \prime} \in \widetilde{T}}{\mathrm{X}[\mathbf{x}] \equiv_{\widetilde{T}} \mathrm{X}\left[\mathbf{x}^{\prime \prime}\right]} \\
& \frac{\mathbf{x}+\mathbf{x}^{\prime}=\mathbf{x}^{\prime \prime} \in \widetilde{T}}{\mathrm{X}[\mathbf{x}] \equiv_{\widetilde{T}} \mathrm{X}\left[\mathbf{x}^{\prime \prime}\right]} \quad \frac{\mathbf{x}+\mathbf{x}^{\prime}=\mathbf{x}^{\prime \prime} \in \widetilde{T}}{\mathrm{X}\left[\mathbf{x}^{\prime}\right] \equiv_{\widetilde{T}} \mathrm{X}\left[\mathbf{x}^{\prime \prime}\right]}
\end{aligned}
$$

\section{D.2 Proof of Proposition C.1}

Essentially we have the same as the proof of Proposition 4.1. Only difference is that we need to use the unique sender condition to ensure that the action $\overline{t_{1}}$ is possible in (B.1) in the proof of Proposition 4.1 (note that $\overline{t_{1}}$ is always possible in basic CFSMs since they are directed).

Suppose, in (B.1) in the proof of Proposition 4.1, the action $\overline{t_{1}}$ is not possible: i.e. $s_{1} \stackrel{t_{1}}{\rightarrow} s_{2} \stackrel{\varphi_{2}}{\longrightarrow} s_{2}^{\prime}$ but $s_{2}^{\prime}$ cannot perform $\stackrel{\overline{t_{1}}}{\rightarrow}$. The only possibility is that some $M_{\mathrm{q}}$ contains the receiver state $q$ such that $\left(q, \mathrm{pq} ? a, q^{\prime}\right),\left(q, \mathrm{p}^{\prime} \mathrm{q} ? b, q^{\prime \prime}\right) \in \delta_{\mathrm{q}}$ which does not satisfy the parallel condition (since if so, $s_{2}^{\prime}$ can perform $\stackrel{\bar{t}_{1}}{\rightarrow}$ ), and $\varphi_{2}$ contains the action $\mathrm{p}^{\prime} \mathrm{q} ? b$, which implies $\varphi_{2}$ contains the action $\mathrm{p}^{\prime} \mathrm{q} ! b$. By the unique sender condition, there is the unique $\mathrm{q}^{\prime}$ such that $s_{0}^{\prime} \stackrel{\varphi \cdot \mathrm{pq} ! a}{\longrightarrow} s_{1}$ and $s_{0}^{\prime} \stackrel{\varphi \cdot \mathrm{pq} ! a \cdot \varphi^{\prime} \cdot \mathrm{p}^{\prime} \mathrm{q} ! b \cdot \varphi^{\prime \prime}}{\longrightarrow} s_{2}^{\prime}$ with $A \operatorname{Send}(\varphi \cdot \mathrm{pq} ! a)=$ 
$A \operatorname{Send}\left(\varphi \cdot \mathrm{pq} ! a \cdot \varphi^{\prime} \cdot \mathrm{p}^{\prime} \mathrm{q} ! b\right)=\left\{\mathrm{q}^{\prime}\right\}$. Since $\mathrm{p}^{\prime} \mathrm{q} ! b$ cannot be reordered before $\mathrm{pq} ! a$ or after $\varphi_{1}$, to satisfy the unique sender property, $\varphi^{\prime}$ should include pq?a. This contradicts that the assumption that $\varphi_{2}$ does not include pq?a.

\section{D.3 Proof of Theorem C.2}

By (reception error freedom) and (orphan message-freedom), together with (stableproperty), we only have to check, there is no input is waiting with an empty queue forever. Suppose by contradiction, there is $s \in R S(S)$ such that $s=(\vec{q} ; \vec{\varepsilon})$ and there exists input state $q_{\mathrm{p}} \in \vec{q}$ and no output transition from $q_{k}$ such that $k \neq \mathrm{q}$.

Then by assumption, there is a 1-buffer execution $\varphi$ and since $\varphi$ is not taken (if so, $q_{\mathrm{p}}$ can perform an input), then there is another execution $\varphi^{\prime}$ such that it leads to state $s$ which is deadlock at $q_{\mathrm{p}}$.

Case (1) Suppose $\varphi$ does not include input actions at q except $a$, i.e. $a$ is the first input action at $q$ in $\varphi$. We let $\varphi_{0}$ for the prefix before the actions of qp! $a \cdot q p$ ? .

By (receiver condition), we know $\mathrm{p} \in \operatorname{Rcv}\left(\varphi^{\prime}\right)$.

By the determinacy, the corresponding input action has a different label from $a$, i.e. $\mathrm{q}^{\prime} \mathrm{p} ? a^{\prime} \in \varphi^{\prime}$. By the diamond property, $\mathrm{q}^{\prime} \mathrm{p} ? a^{\prime}$ and $\mathrm{qp} ? a$ can be appeared from the same state, i.e. this state is under the assumption of the parallel condition. Hence by the multiparty compatibility, the both corresponding outputs $\mathrm{q}^{\prime} \mathrm{p} ! a^{\prime}$ and $\mathrm{qp} ! a$ can be always fired if one of them is. This contradicts the assumption that $q_{\mathrm{p}}$ is deadlock with label $a$.

Case (2) Suppose $\varphi$ includes other input actions at $\mathrm{q}$ before $\mathrm{qp}$ ? $a$, i.e. $\mathrm{p} \in \operatorname{Rcv}\left(\varphi_{0}\right)$. Let $\mathrm{q}^{\prime} \mathrm{p} ? a^{\prime}$ the action which first occurs in $\varphi_{0}$. By $\mathrm{p} \in \operatorname{Rcv}\left(\varphi^{\prime}\right)$, there exists $\mathrm{q}^{\prime \prime} \mathrm{p} ? a^{\prime \prime} \in$ $\varphi^{\prime}$. If $\mathrm{q}^{\prime \prime} \mathrm{p} ? a^{\prime \prime} \neq \mathrm{q}^{\prime} \mathrm{p} ? a^{\prime}$, by the same reasoning as $(1)$, the both corresponding outputs are available. Hence we assume the case $\mathrm{q}^{\prime \prime} \mathrm{p} ? a^{\prime \prime}=\mathrm{q}^{\prime} \mathrm{p} ? a^{\prime}$. Let $s$ is the first state from which a transition in $\varphi_{0}$ and a transition in $\varphi^{\prime}$ are separated. Then by assumption, if $s \stackrel{\varphi_{0} \cdot q^{\prime} \mathrm{p} ! a^{\prime} \cdot \mathrm{q}^{\prime} \mathrm{p} ? a^{\prime}}{\longrightarrow} s_{1}$ and $s \stackrel{\varphi_{1} \cdot \mathrm{q}^{\prime} \mathrm{p} ! a^{\prime} \cdot \mathrm{q}^{\prime} \mathrm{p} ? a^{\prime}}{\longrightarrow} s_{2}$, by assumption $a^{\prime} \notin \varphi_{0} \cup \varphi_{1}$, hence $s \stackrel{\mathrm{q}^{\prime} \mathrm{p} ! a^{\prime} \cdot \mathrm{q}^{\prime} \mathrm{p} ? a^{\prime}}{\longrightarrow} s_{1}^{\prime} \stackrel{\varphi_{0}^{\prime}}{\longrightarrow} s_{1}$ and $s \stackrel{\mathrm{q}^{\prime} \mathrm{p} ! a^{\prime} \cdot \mathrm{q}^{\prime} \mathrm{p} ? a^{\prime}}{\longrightarrow} s_{2}^{\prime} \stackrel{\varphi_{1}^{\prime}}{\longrightarrow} s_{2}$ by the diamond property again. Since $s_{1}$ can perform an input at q by the assumption (because of qp?a), $\varphi_{1}^{\prime}$ should contain an input at $\mathrm{q}$ by the receiver condition. If it contains the input to $\mathrm{q}$ in $\varphi_{1}^{\prime}$, then we repeat Case (2) noting that the length of $\varphi_{1}^{\prime}$ is shorter than the length of $\varphi_{1} \cdot \mathrm{q}^{\prime} \mathrm{p} ! a^{\prime} \cdot \mathrm{q}^{\prime} \mathrm{p} ? a^{\prime}$; else we use Case (1) to lead the contradiction; otherwise if it contains the same input as $\mathrm{qp} ? a$, then it contradicts the assumption that $q_{\mathrm{p}}$ is deadlock. 